\title{
Chinese Medicinal Herbs for Childhood Pneumonia: A Systematic Review of Effectiveness and Safety
}

\author{
Qianchun Yang, ${ }^{1,2}$ Darong Wu, ${ }^{3}$ Wei Mao, ${ }^{4}$ Xusheng Liu, ${ }^{2}$ Kun Bao, ${ }^{2}$ Qizhan Lin, \\ Fuhua $\mathrm{Lu}^{2}{ }^{2}$ Chuan $\mathrm{Zou},{ }^{2}$ and Chuang $\mathrm{Li}^{4}$ \\ ${ }^{1}$ The Second Clinical Medical College, Guangzhou University of Chinese Medicine, Guangzhou 510405, China \\ ${ }^{2}$ Department of Nephropathy Medicine, Guangdong Provincial Hospital of Chinese Medicine, Guangzhou 510120, China \\ ${ }^{3}$ Outcome Assessment Branch, Department of Clinical Epidemiology, Guangdong Provincial Hospital of Chinese Medicine, \\ Guangzhou 510120, China \\ ${ }^{4}$ Department of Nephrology laboratory, Guangdong Provincial Hospital of Chinese Medicine, Guangzhou 510120, China
}

Correspondence should be addressed to Wei Mao; maowei1274@126.com

Received 12 August 2012; Revised 6 November 2012; Accepted 15 November 2012

Academic Editor: Zhao Xiang Bian

Copyright (C) 2013 Qianchun Yang et al. This is an open access article distributed under the Creative Commons Attribution License, which permits unrestricted use, distribution, and reproduction in any medium, provided the original work is properly cited.

Objective. To assess the efficacy and safety of Chinese medicinal herbs for Childhood Pneumonia. Methods. We included randomized controlled trials (RCTs). The searched electronic databases included PubMed, the Cochrane Central Register of Controlled Trials, EMBASE, CBM, CNKI, and VIP. All studies included were assessed for quality and risk bias. Review Manager 5.1.6 software was used for data analyses, and the GRADEprofiler software was applied to classify the systematic review results. Results. Fourteen studies were identified $(n=1.824)$. Chinese herbs may increase total effective rate (risk ratio (RR) 1.18 ; $95 \%$ confidence interval (CI), 1.11-1.26) and improve cough (total mean difference (MD), -2.18 ; 95\% CI, (-2.66) $-(-1.71)$ ), fever (total MD, -1.85 ; 95\% $\mathrm{CI},(-2.29)-(-1.40)$ ), rales (total MD, -1.53 ; 95\% CI, $(-1.84)-(-1.23)$ ), and chest films (total MD, -3.10 ; 95\% CI, $(-4.11)-(-2.08)$ ) in Childhood Pneumonia. Chinese herbs may shorten the length of hospital stay (total MD, -3.00 ; 95\% CI, (-3.52) $-(-2.48)$ ), but no significant difference for adverse effects (RR, 0.39; 95\% CI, 0.09-1.72) was identified. Conclusion. Chinese herbs may increase total effective rate and improve symptoms and signs. However, large, properly randomized, placebo-controlled, double-blind studies are required.

\section{Introduction}

Childhood Pneumonia is an acute virus, bacterial, or fungal respiratory infection that affects the lungs [1]. The symptoms and signs of Childhood Pneumonia include cough, fever, rapid or difficult breathing, loss of appetite, and lower chest wall indrawing [2]. Auscultation reveals rales. However, severe Childhood Pneumonia is defined as cough or difficult breathing combined with lower chest wall indrawing [3]. Childhood Pneumonia has been identified as a mixed viralbacterial infection in $23 \%-33 \%$ of cases [4]. Respiratory syncytial virus is the most common viral cause of children pneumonia, and Streptococcus pneumoniae is the most common cause of bacterial pneumonia in children [5]. Each year, pneumonia kills an estimated 1.4 million children $<5$ years of age, accounting for $18 \%$ of all deaths worldwide [6].
Pneumonia is the most common reason for hospitalization in children $<2$ years of age [7]. The cost of antibiotic treatment for all children with pneumonia in 42 of the poorest countries is estimated to be about US $\$ 600$ million per year. The estimated incidence rates are 0.29 and 0.05 episodes per childyear in low-income and high-income countries, respectively. Approximately 156 million new episodes occur each year, the majority in India (43 million), China (21 million), Pakistan (10 million), Bangladesh, Indonesia, and Nigeria (6 million each) [8].

Administering appropriate antibiotics at the early stage of pneumonia improves outcomes, particularly when the causative agent is bacterial [9]. However, antibiotic treatment of pneumonia in children remains mostly empirical because determining the etiologic pathogen is difficult in this age group [10]. According to the results of a questionnaire, 
the following agents have been used against Childhood Pneumonia: ampicillin, ampicillin/sulbactam, second/third generation cephalosporins, azithromycin, vancomycin, clindamycin, and linezolid. In subsequent analyses, we categorized ampicillin, ampicillin/sulbactam, and cephalosporins as beta-lactam antibiotics and vancomycin, clindamycin, and linezolid as antimethicillin-resistant Staphylococcus aureus antibiotics. Respondents were asked to select the duration of antibiotic therapy they would recommend for uncomplicated and parapneumonic empyema cases using the following categories: 3-5 days, 6-7 days, 8-10 days, 11-14 days, 15-21 days, and $>21$ days [11]. Empirical antibiotic administration is relied upon in most instances to meet the public health goal of reducing child mortality due to pneumonia. This is necessary in view of the inability of most commonly available laboratory tests to identify causative pathogens. Empirical antibiotic administration is the main treatment for Childhood Pneumonia. Multiple antibiotics are prescribed for treating pneumonia, so it is important to know which work best for pneumonia in children [12].

Traditional Chinese Medicine (TCM) follows a particular theoretical and methodological approach to estimate the cause of a disease, leading to diagnosis and treatment [13]. Pneumonia is equivalent to the TCM cough category. Ma Xing Shi Gan Tang, San Ao Tang, Zhi Sou San, and other self-developed TCM prescriptions are Chinese medicinal formulas that have been used to treat Childhood Pneumonia for many years. In recent years, preparations of Chinese herbal medicines, such as Tanreqing injection, Chuanhuning injection, and Reduning injection have been used to treat Childhood Pneumonia in China. Chinese herbal medicine formulas function to clear heat, resolve phlegm, ventilate the lungs, dissipate phlegm, relieve cough, and reduce sputum. The function of Chinese herbal medicine preparations is to clear heat and remove toxicity. A study showed that the pharmacological action of Ma Xing Shi Gan Tang includes antiasthmatic, antitussive, and antiviral effects as well as bacteriostatic and immunoregulatory functions [14].

Although these formulae and other Chinese herbal medicine preparations have been used widely to treat Childhood Pneumonia in China, their effects and safety have not been reviewed systematically.

\section{Methods}

\subsection{Criteria for Considering Studies for this Paper}

2.1.1. Types of Studies. Only randomized controlled trials (RCTs) were included.

2.1.2. Types of Participants. Studies that enrolled patients with pneumonia in children who had cough, fever $>37.5^{\circ} \mathrm{C}$, raised respiratory rate, lower chest wall indrawing, rales, and changes on chest films were included. Patients with Childhood Pneumonia of either gender, any ethnic group, and ages of 1 month to 18 years were included. Studies were excluded if they included children suffering from other debilitating diseases.
2.1.3. Types of Interventions. There were Chinese medicinal herbs versus other drugs, formulas, and placebo alone; Chinese medicinal herbs plus basic therapy versus basic therapy. Antibiotics were one of the main basic therapies for Childhood Pneumonia. Prohibited or suspended Chinese herbal preparations were excluded.

2.1.4. Types of Outcome Measures. Primary outcomes included mortality and total effective rate (e.g., ratio of signs and symptoms improvement or recovery); secondary outcomes included time to clinical recovery (e.g., cough, fever, rales, and chest films), relapse rate, length of hospital stay, and adverse effects (e.g., nausea, diarrhea, vomiting, and gastrointestinal bleeding). TCM outcomes such as tongue coat, pulse condition, and economic index were included.

2.2. Search Methods for Identification Studies. We searched for all relevant studies in the following electronic databases: PubMed (1966-July 2012), the Cochrane Central Register of Controlled Trials, EMBASE (1980-July 2012), the Chinese Biomedicine Database (CBM) (1976-July 2012), Chinese National Knowledge Internet (CNKI) (1979-July 2012), and Chinese Biomedical Journals (VIP) (1989-July 2012). All studies included were analyzed according to Cochrane Handbook criteria. The following search terms were used: (Chinese herbs OR Chinese traditional herbs OR Chinese medicinal herbs OR traditional Chinese herbs OR Chinese herbal medicines) AND (child pneumonia OR children pneumonia OR Childhood Pneumonia OR Pediatric Pneumonia OR Infantile Pneumonia). We conducted a manual search for the Journal of Guangzhou University of Traditional Chinese Medicine. We attempted to contact original authors to obtain the protocol for the studies.

\subsection{Data Collection and Analysis}

2.3.1. Study Selection. Two review authors independently browsed the titles and abstracts of all articles identified by the literature search. The same review authors independently estimated whether the trials met the inclusion criteria. Disagreements were resolved by discussion or consultation with a third author. We assessed abstracts from the initial search independently to identify studies that met the inclusion criteria. We telephone-interviewed authors of Chinese language articles and emailed the original authors of English articles to identify the randomization procedure and other methodological questions to ensure that the included studies were RCTs. If the required information was not available or if the required information did not meet the inclusion criteria, the article was excluded.

2.3.2. Data Extraction and Management. We extracted data including methodological details and data from publications using a data extraction form. We extracted data on study characteristics, including methods, participants, interventions, and outcomes. There were no disagreements among the authors. 
TABLE 1: Contents of the formulations used and the three languages are included in the included studies.

\begin{tabular}{lll}
\hline Study ID & Herbs (composition) in three languages & Method of administration \\
\hline & $\begin{array}{l}\text { Modified Ma Xing Shi Gan Tang: Mahuang (Herba Ephedrae/Ephedra Herb), Xingren } \\
\text { (Armeniacae Amarum/Bitter Apricot Seed), Shigao (Gypsum Fibrosum/Gypsum), }\end{array}$ \\
Wang et al., 2009 [15] & $\begin{array}{l}\text { Gancao (Radix Glycyrrhizae/Liquorice Root), Yuxingcao (Herba Houttuyniae/Heartleaf } \\
\text { Houttuynia Herb), lianqiao (Fructus Forsythiae/WeepingForsythiae capsule), Chanyi } \\
\text { (Periostracum Cicadae/Cicada Slough), and Niupangzi (Fructus Arctii/ Great Burdock }\end{array}$ \\
& Achene) & Oral administration \\
& $\begin{array}{l}\text { Modified Ma Xing Shi Gan Tang: Mahuang (Herba Ephedrae/Ephedra Herb), Xingren } \\
\text { (Armeniacae Amarum/Bitter Apricot Seed), Shigao (Gypsum Fibrosum/Gypsum), }\end{array}$ \\
& $\begin{array}{l}\text { Gancao (Radix Glycyrrhizae/Liquorice Root), Yuxingcao (Herba Houttuyniae/Heartleaf } \\
\text { Zhao and Ji, 2009 [16] Oral administration }\end{array}$ \\
& Houttuynia Herb), and Huangqin (Radix Astragali Root) \\
\hline Gudified Ma Xing Shi Gan Tang: Mahuang (Herba Ephedrae/Ephedra Herb), Xingren \\
(Armeniacae Amarum/Bitter Apricot Seed), Shigao (Gypsum Fibrosum/Gypsum), \\
Gancao (Radix Glycyrrhizae/Liquorice Root), Yuxingcao (Herba Houttuyniae/Heartleaf \\
Houttuynia Herb), and lianqiao (Fructus Forsythiae/WeepingForsythiae Capsule)
\end{tabular}
Houttuynia Herb), and lianqiao (Fructus Forsythiae/WeepingForsythiae Capsule)

Modified Ma Xing Shi Gan Tang: Mahuang (Herba Ephedrae/Ephedra Herb), Xingren

Zhang, 2012 [18] (Armeniacae Amarum/Bitter Apricot Seed), Shigao (Gypsum Fibrosum/Gypsum), Gancao (Radix Glycyrrhizae/Liquorice Root), Yuxingcao (Herba Houttuyniae/Heartleaf

Oral administration Houttuynia Herb), and Huangqin (Radix Astragali Root)

Modified Ma Xing Shi Gan Tang: Mahuang (Herba Ephedrae/Ephedra Herb), Xingren (Armeniacae Amarum/Bitter Apricot Seed), Jinhua (Flos Lonicerae/Honeysuckle

He, 2011 [19] Flower), Yinhua (Flos Lonicerae/Honeysuckle Flower), Shigao (Gypsum

Oral administration Fibrosum/Gypsum), Yuxingcao (Herba Houttuyniae/Heartleaf Houttuynia Herb), Banxia (Rhizome/Pinellia Tuberifera Tenora), and Gancao (Radix Glycyrrhizae/Liquorice Root)

Modified Ma Xing Shi Gan Tang: Mahuang (Herba Ephedrae/Ephedra Herb), Xingren (Armeniacae Amarum/Bitter Apricot Seed), Shigao (Gypsum Fibrosum/Gypsum), Suzi (Fructus Perillae/PerillaFruit), Shangbaipi (Cortex Mori/White Mulberry Root Bark),

He et al., 2011 [20] Kuandonghua (Flos Farfarae/Common Coltsfoot Flower), Banxia (Rhizome/Pinellia Oral administration Tuberifera Tenora), Tinglizi (Semen Lepidii/SemenDescurainiae Pepperweed Seed/Tansymustard), Yuxingcao (Herba Houttuyniae/Heartleaf Houttuynia Herb), and Gancao (Radix Glycyrrhizae/Liquorice Root)

Zhang, 2012 [21] San Ao Tang: Mahuang (Herba Ephedrae/Ephedra Herb), Xingren (Armeniacae Amarum/Bitter Apricot Seed), and Gancao (Radix Glycyrrhizae/Liquorice Root)

Oral administration

Modified Zhi Sou San: Jiegeng (Radix Platycodi/Platycodon Root), Gancao (Radix Glycyrrhizae/Liquorice Root), Ziwan (Radix Asteris/Tatarian Aster Root), Chenpi

Wang et al., 2009 [22] (Pericarpium Citri Reticulatae/Tangerine Peel), Xingren (Armeniacae Amarum/Bitter Apricot Seed), Baiguo (Semen Gingko/Ginkgo Seed), Huangqi (Radix Astragali Root),

Oral administration Chaomaiya (Fructus Hordei Germina/Malt), Yunling (Poria/Indian Buead), and Baiqian (Rhizoma Cynanchi Stauntonii/Willowleaf Swallowwort Rhizome/Glaucescent)

Self-Developed TCM Prescription: Mahuang (Herba Ephedrae/Ephedra Herb), Xingren (Armeniacae Amarum/Bitter Apricot Seed), Rengongniuhuang (Calculus Bovis/Bezoar), Baiqian (Rhizoma Cynanchi Stauntonii/Willowleaf Swallowwort Rhizome/Glaucescent), Shigao (Gypsum Fibrosum/Gypsum), Zhusha (Cinnabaris/Cinnabar), Chuanbeimu

Lv et al., 2009 [23]

(Bulbus Fritillariae Unibracteatae/Unibract Fritillary Bulb), Huanglian (Rhizoma

Oral administration Coptidis/Golden Thread), Banxia (Rhizome/Pinellia Tuberifera Tenora), Dannanxing (PinelliaPedatisecta /Arisaema with Bile), Shangbaipi (Cortex Mori/White Mulberry Root Bark), Huangqin (Radix Astragali Root), and Gancao (Radix Glycyrrhizae/Liquorice Root)

\begin{tabular}{lccc}
\hline Lei, $2010[24]$ & Tanreqing injection: no information provided about Tanreqing composition & Intravenous injection \\
\hline Shi, 2009 [25] & Reduning injection: no information provided about Reduning composition & Intravenous injection \\
\hline Pan, 2011 [26] & Reduning injection: no information provided about Reduning composition & Intravenous injection \\
\hline Duan and Feng, 2011 [27] Reduning injection: no information provided about Reduning composition & Intravenous injection \\
\hline Wei and Feng, 2003 [28] Chuanhuning injection: no information provided about Chuanhuning composition & Intravenous injection \\
\hline
\end{tabular}


We extracted the formulation contents of the included studies, and the names of the herbs are provided in three languages (e.g., Chinese, Latin, and English) in Table 1.

2.3.3. Assessment of Risk of Bias in Included Studies. The following items were independently assessed by our authors using the risk of bias assessment tool. (1) Was there adequate sequence generation (selection bias)? (2) Was allocation adequately concealed (selection bias)? (3) Was knowledge of the allocated interventions adequately prevented during the study (e.g., participants and personnel, outcome assessors) (detection bias)? (4) Were incomplete outcome data adequately addressed (attrition bias)? (5) Are reports of the study free of suggesting selective outcome reporting (reporting bias)? (6) Was the study apparently free of other problems that could put it at risk for bias?

2.3.4. Measures of Treatment Effect. Data analyses were performed using the Cochrane Collaboration's RevMan software, version 5.1.6. Results are expressed as risk ratios (RR) and 95\% confidence intervals (CIs) for dichotomous outcomes (e.g., mortality, effective rate, adverse effects, and relapse rate) and as mean differences (MD) with 95\% CIs for continuous outcomes (such as time to clinical recovery and length of hospital stay).

2.3.5. Assessment of Heterogeneity. Heterogeneity was analyzed using the chi-square test on $n-1$ degrees of freedom, and an alpha of 0.05 was used for statistical significance with the $I^{2}$ test. $I^{2}$ values of 25,50 , and $75 \%$ corresponded to low, medium, and high levels of heterogeneity, respectively.

2.3.6. Data Synthesis. We used fixed-effects and randomeffects models for the pooled data analysis. We performed a pooled analysis for the 14 studies.

2.3.7. Subgroup Analyses. Subgroup analyses were performed according to formula type and Chinese medicinal herb preparation type, using the same comparators (e.g., same types of antibiotics).

2.3.8. Sensitivity Analyses. Sensitivity analyses were conducted by excluding low-quality studies (based on descriptions of randomization, allocation concealment, blinded assessment of outcomes, and description/analyses of withdrawals and dropouts) and a comparison of the merger analysis results for the fixed- and random-effects models.

\section{Results}

3.1. Search Results. An initial search identified 2,502 potentially relevant articles. Of these, 15 were in the English database. A total of 891 articles were initially included after duplicate publications were removed and any obviously irrelevant were excluded; 800 articles were later excluded, because they did not meet the inclusion criteria. Of the 91 potentially eligible reports, 77 were excluded for further assessment because telephone interviews with the original authors revealed that they were not RCTs. Therefore, 14 studies (1,824 participants) were included in this paper. All 14 studies were published in Chinese (Figure 1).

\subsection{Included Studies}

3.2.1. Participants. The ratio of male to female participants in the 14 studies was $879 / 615$ [15-21, 23-28]. One study [22] did not report the number of males and females. In total, 1,824 children were included in the 14 studies, and all were from China [15-28]. The ages of the patients were 1 month-15 years. The average size of the trials was 130 participants (range 60200 participants).

3.2.2. Inclusion Criteria. The diagnostic criteria for childhood pneumonia in all studies included fever $>37.5^{\circ} \mathrm{C}$, chest recession, increased respiratory rate, cough, rales, or difficulty breathing combined with fast breathing and a change on chest films.

3.2.3. Intervention. Chinese medicinal herbs interventions were given as oral decoctions or intravenous infusions. The longest therapy duration was 3 weeks, and the shortest was 5 days. Follow-up duration was not mentioned by any of the authors. All 14 studies compared Chinese medicinal herbs plus basic therapy versus basic therapies.

3.2.4. Outcomes. All 14 studies [15-28] reported the total effective rate; seven studies $[15,17,18,21,23,25,28]$ reported clinical recovery of cough, fever, rales, and chest films; four $[16,22,24,26]$ reported clinical recovery of cough, fever, and rales; one [27] reported clinical recovery of fever; three [15, 21, 22] reported adverse effects (e.g., nausea, vomiting, diarrhea, and gastrointestinal bleeding); and one [28] reported the length of hospital stay. The description of studies is detailed in the characteristics of included studies in Table 2.

3.3. Risk of Bias in Included Studies. The methodological quality of each study's randomization sequence, allocation concealment, blinding, incomplete outcome data, selective outcome reporting, and potential threats are summarized in Figures 2 and 3.

3.3.1. Randomization and Allocation Concealment. Ten studies [15-20, 23, 25-27] reported using a random-number table, and four $[21,22,24,28]$ reported using a computer-generated random-number table. None of the trials used allocation concealment. Therefore, all studies had a high risk of selection bias.

3.3.2. Blinding. Ten studies [15-17, 20-24, 26, 27] used single blinding (outcome assessment was blinded). We interviewed the original authors by telephone to determine blinding because the blinding methods were not described. Thus, these studies had a low risk of performance bias and low detection bias. The other studies $[18,19,25,28]$ did not use blinding 


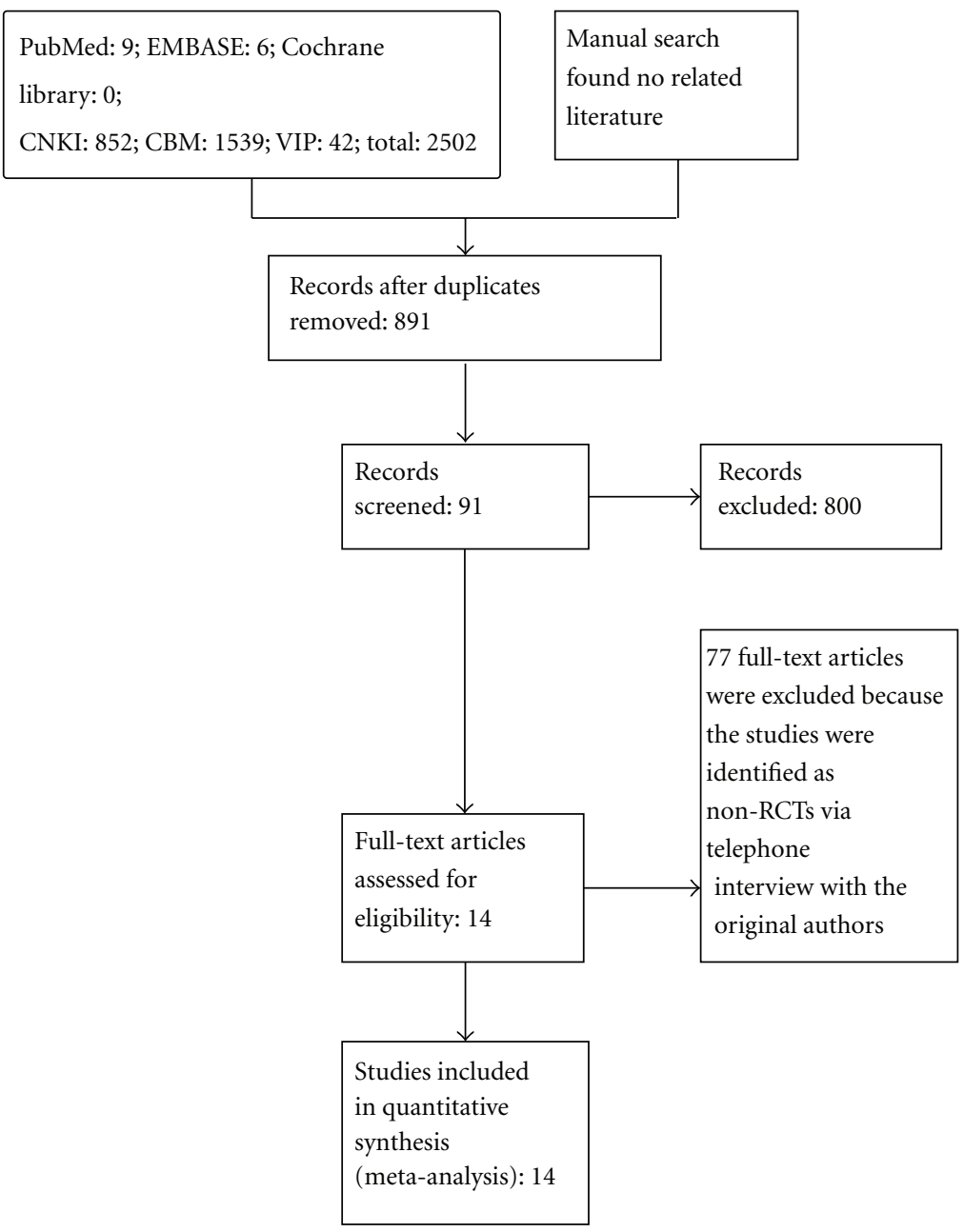

FIGURE 1: Summary of the search results in a flow diagram.

Random sequence generation (selection bias)

Allocation concealment (selection bias)

Blinding of participants and personnel (performance bias) Blinding of outcome assessment (detection bias) Incomplete outcome data (attrition bias) Selective reporting (reporting bias)

Other bias

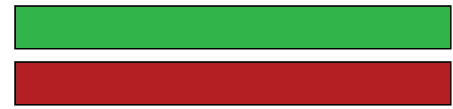

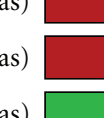
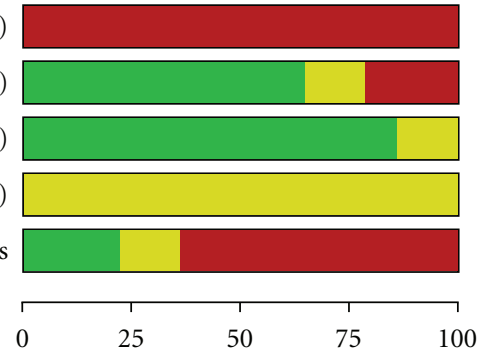

(\%)

$\square$ Low risk of bias

$\square$ Unclear risk of bias

$\square$ High risk of bias

FIGURE 2: Methodological quality. Judgments about each item are presented as percentages across all included studies. 
TABLE 2: Characteristics of the included studies.

\begin{tabular}{|c|c|}
\hline & Wang et al., 2009 [15] \\
\hline \multirow{8}{*}{ Methods } & Randomized controlled trial (RCT): randomization mentioned, but not described in detail \\
\hline & Allocation concealment: not mentioned \\
\hline & Followup: not mentioned \\
\hline & Study duration: 3 weeks \\
\hline & Parallel/crossover/factorial RCT: parallel \\
\hline & $\begin{array}{l}\text { Randomization method: we interviewed the author by telephone and learned that a random number table was } \\
\text { used to generate the random sequence }\end{array}$ \\
\hline & $\begin{array}{l}\text { Blinding: no detailed information on blindness was offered. A telephone interview with the author revealed that } \\
\text { single blinding was used }\end{array}$ \\
\hline & ITT: not mentioned \\
\hline \multirow{4}{*}{ Participants } & Setting: inpatients \\
\hline & Country: China \\
\hline & Number: 106 patients with childhood pneumonia \\
\hline & 54 boys (50.8\%) and 52 girls (49.2\%); age 3-14 years old; disease duration: not mentioned \\
\hline \multirow[t]{2}{*}{ Interventions } & $\begin{array}{l}\text { Treatment group: modified Ma Xing Shi Gan Tang formula plus basic therapy: Mahuang } 6 \mathrm{~g} \text {, Xingren } 8 \mathrm{~g} \text {, } \\
\text { Shigao } 15 \mathrm{~g} \text {, Gancao } 5 \mathrm{~g} \text {, Yuxingcao } 20 \mathrm{~g} \text {, lianqiao } 15 \mathrm{~g} \text {, Chanyi } 10 \mathrm{~g} \text {, and Niupangzi } 15 \mathrm{~g} \text { boiled in } 3 \mathrm{~L} \text { water and } \\
\text { decocted to } 300 \mathrm{~mL} \text {. Orally twice daily (bid) for } 3 \text { weeks }\end{array}$ \\
\hline & Control group: basic therapy including intravenous infusion of azithromycin and azithromycin orally \\
\hline \multirow{2}{*}{ Outcomes } & (1) Total effective rate \\
\hline & (2) Adverse effects (e.g., nausea, diarrhea, and vomit) \\
\hline Notes & $\begin{array}{l}\text { (1) Duration of disease: not mentioned; (2) mortality: not mentioned; (3) relapse rate: not mentioned; (4) length } \\
\text { of hospital stay: not mentioned; (5) clinical recovery (e.g., cough, fever, rales, and chest films): not mentioned; } \\
\text { (6) TCM outcomes, such as tongue coat and pulse condition: not mentioned; (7) economic index: not } \\
\text { mentioned; (8) withdrawal rates: not specified; (9) source of funding: none }\end{array}$ \\
\hline \multicolumn{2}{|r|}{ Zhao and Ji, 2009 [16] } \\
\hline \multirow{7}{*}{ Methods } & RCT: randomization mentioned, but not described in detail \\
\hline & Allocation concealment: not mentioned \\
\hline & Followup was not mentioned \\
\hline & Study duration: not mentioned \\
\hline & Parallel/crossover/factorial RCT: parallel \\
\hline & $\begin{array}{l}\text { Randomization method: a telephone interview with the author revealed that a random number table was used } \\
\text { to generate the random sequence }\end{array}$ \\
\hline & $\begin{array}{l}\text { Blinding: no detailed information on blindness was offered. A telephone interview with the author revealed that } \\
\text { single blinding was used }\end{array}$ \\
\hline \multirow{6}{*}{ Participants } & ITT: not mentioned \\
\hline & Setting: inpatients and outpatients \\
\hline & Country: China \\
\hline & Number: 60 patients with childhood pneumonia \\
\hline & $\begin{array}{l}\text { Treatment group: } 30 \text { patients with childhood pneumonia: } 16 \text { boys ( } 53 \%) \text { and } 14 \text { girls ( } 47 \%) \text {; age: } 2 \text { months }-9 \\
\text { years (mean: } 3.5 \text { years); disease duration: } 4.00 \pm 1.55 \text { years }\end{array}$ \\
\hline & $\begin{array}{l}\text { Control group: } 30 \text { patients with childhood pneumonia: } 17 \text { boys }(56.6 \%) \text { and } 13 \text { girls ( } 43.4 \%) \text {; age:month }-11 \\
\text { years (mean: } 3.25 \text { years); disease duration: } 4.00 \pm 1.75 \text { years }\end{array}$ \\
\hline \multirow[t]{2}{*}{ Interventions } & $\begin{array}{l}\text { Treatment group: modified Ma Xing Shi Gan Tang formula plus basic therapy: Mahuang } 6 \mathrm{~g} \text {, Xingren } 10 \mathrm{~g} \text {, } \\
\text { Shigao } 20 \mathrm{~g} \text {, Gancao } 3 \mathrm{~g} \text {, Yuxingcao } 10 \mathrm{~g} \text {, and Huangqin } 10 \mathrm{~g} \text { boiled in } 3 \mathrm{~L} \text { water and decocted to } 300 \mathrm{~mL} \text {. Orally } \\
\text { twice daily (bid) for } 3 \text { weeks }\end{array}$ \\
\hline & Control group: basic therapy including intravenous infusion of azithromycin and azithromycin orally \\
\hline \multirow{2}{*}{ Outcomes } & (1) Total effective rate \\
\hline & (2) Clinical recovery (e.g., cough, fever, and rales) \\
\hline Notes & $\begin{array}{l}\text { (1) Mortality: not mentioned; (2) relapse rate: not mentioned; (3) length of hospital stay: not mentioned; (4) } \\
\text { TCM outcomes, such as tongue coat and pulse condition: not mentioned; (5) clinical recovery (e.g., chest films): } \\
\text { not mentioned; (6) economic index: not mentioned; (7) withdrawal rates: not mentioned; (8) source of funding: } \\
\text { none;(9) adverse effects (e.g., nausea, diarrhea, and vomiting): not mentioned }\end{array}$ \\
\hline
\end{tabular}


TABLe 2: Continued.

\begin{tabular}{|c|c|}
\hline & Guo, 1999 [17] \\
\hline \multirow{7}{*}{ Methods } & RCT: randomization mentioned, but not described in detail \\
\hline & Allocation concealment: not mentioned \\
\hline & Followup: not mentioned \\
\hline & Not mentioned \\
\hline & Parallel/crossover/factorial RCT: parallel \\
\hline & $\begin{array}{l}\text { Randomization method: a telephone interview with the author revealed that a random number table was used } \\
\text { to generate the random sequence }\end{array}$ \\
\hline & $\begin{array}{l}\text { Blinding: no detailed information on blindness was offered. A telephone interview with the author revealed that } \\
\text { single-blinding was used }\end{array}$ \\
\hline \multirow{6}{*}{ Participants } & ITT: not mentioned \\
\hline & Setting: not mentioned \\
\hline & Country: China \\
\hline & Number: 170 patients with childhood pneumonia \\
\hline & $\begin{array}{l}\text { Treatment group: } 86 \text { patients with childhood pneumonia: } 45 \text { boys }(52.3 \%) \text { and } 41 \text { girls ( } 48.7 \%) \text {; age: } 2 \\
\text { months-12 years (mean: } 3.8 \text { years) }\end{array}$ \\
\hline & $\begin{array}{l}\text { Control group: } 84 \text { patients with childhood pneumonia: } 44 \text { boys }(52.4 \%) \text { and } 40 \text { girls ( } 48.6 \%) \text {; age: } 2 \text { months-14 } \\
\text { years (mean: } 3.2 \text { years) }\end{array}$ \\
\hline \multirow[t]{2}{*}{ Interventions } & $\begin{array}{l}\text { Treatment group: modified Ma Xing Shi Gan Tang formula plus basic therapy. Mahuang } 1.5 \mathrm{~g} \text {, Xingren } 3 \mathrm{~g} \text {, } \\
\text { Shigao } 10 \mathrm{~g} \text {, Gancao } 1.5 \mathrm{~g} \text {, Yuxingcao } 9 \mathrm{~g} \text {, and lianqiao } 3 \mathrm{~g} \text {, boiled in } 3 \mathrm{~L} \text { water and decocted to } 300 \mathrm{~mL} \text {. Taken } \\
\text { orally, three times daily (tid) }\end{array}$ \\
\hline & $\begin{array}{l}\text { Control group: basic therapy included penicillin,Xianfeng Meisu, and ribavirin. Intravenous infusion of } \\
\text { azithromycin and azithromycin orally }\end{array}$ \\
\hline Outcomes & (1) Total effective rate \\
\hline Notes & $\begin{array}{l}\text { (1) Mortality: not mentioned; (2) relapse rate: not mentioned; (3) length of hospital stay: not mentioned; (4) } \\
\text { TCM outcomes, such as tongue coat and pulse condition: not mentioned; (5) economic index: not mentioned; } \\
\text { (6) withdrawal rates: not specified; (7) source of funding: None; (8) time to measure outcomes: not mentioned; } \\
\text { (9)2. clinical recovery (e.g., cough, fever, rales, and chest films): not mentioned }\end{array}$ \\
\hline \multicolumn{2}{|r|}{ Zhang, 2012 [18] } \\
\hline \multirow{7}{*}{ Methods } & RCT: randomization mentioned, but not described in detail \\
\hline & Allocation concealment: not mentioned \\
\hline & Followup: not mentioned \\
\hline & Study duration: 20 days \\
\hline & Parallel/crossover/factorial RCT: parallel \\
\hline & $\begin{array}{l}\text { Randomization method: a telephone interview with the author revealed that a random number table was used } \\
\text { to generate the random sequence }\end{array}$ \\
\hline & $\begin{array}{l}\text { Blinding: no detailed information on blindness was offered. Telephone interview with author revealed that } \\
\text { blinding was not used }\end{array}$ \\
\hline \multirow{6}{*}{ Participants } & ITT: not mentioned \\
\hline & Setting: not mentioned \\
\hline & Country: China \\
\hline & Number: 200 patients with childhood pneumonia \\
\hline & $\begin{array}{l}\text { Treatment group: } 100 \text { patients with childhood pneumonia: } 74 \text { boys ( } 74 \%) \text { and } 26 \text { girls ( } 47 \%) \text {; mean age: } 6.28 \\
\text { years; disease duration: not mentioned }\end{array}$ \\
\hline & $\begin{array}{l}\text { Control group: } 100 \text { patients with childhood pneumonia: } 68 \text { boys }(68 \%) \text { and } 32 \text { girls ( } 32 \%) \text {; age and duration of } \\
\text { disease not mentioned }\end{array}$ \\
\hline \multirow[t]{2}{*}{ Interventions } & $\begin{array}{l}\text { Treatment group: modified Ma Xing Shi Gan Tang formula plus basic therapy. Mahuang } 3 \mathrm{~g} \text {, Xingren } 4 \mathrm{~g} \text {, } \\
\text { Shigao } 18 \mathrm{~g} \text {, Gancao } 3 \mathrm{~g} \text {, Yuxingcao } 9 \mathrm{~g} \text {, and Huangqin } 3 \mathrm{~g} \text { boiled in } 3 \mathrm{~L} \text { water and decocted to } 300 \mathrm{~mL} \text {, taken } \\
\text { orally twice daily (bid) }\end{array}$ \\
\hline & Control group: basic therapy included intravenous infusion of azithromycin and azithromycin orally \\
\hline \multirow{2}{*}{ Outcomes } & (1) Total effective rate \\
\hline & (2) Clinical recovery (e.g., cough, fever, rales, and chest films) \\
\hline
\end{tabular}


TABle 2: Continued.

(1) Mortality: not mentioned; (2) relapse rate: not mentioned; (3) length of hospital stay: not mentioned; (4)

Notes TCM outcomes, such as the tongue coat and pulse condition: not mentioned; (5) economic index: not mentioned; (6) withdrawal rates: not mentioned; (7) source of funding: none; (8) time to measure outcomes:

not mentioned; (9) adverse effects: not mentioned

$$
\text { He, } 2011[19]
$$

RCT: randomization mentioned, but not described in detail

Allocation concealment: not mentioned

Followup: not mentioned

Methods

Study duration: 5-7 days

Parallel/crossover/factorial RCT: parallel

Randomization method: a telephone interview with the author revealed that a random number table was used to generate the random sequence

Blinding: no detailed information on blindness was offered. A telephone interview with the author revealed that blinding was not used

ITT: not mentioned

Setting: inpatients

Country: China

Participants Number: 100 patients with childhood pneumonia

Treatment group: 50 patients with childhood pneumonia: 29 boys (54\%) and 21 girls (42\%); age: 9.6 months-12 years; disease duration: $4-8.5$ days

Control group: 50 patients with childhood pneumonia: 27 boys (54\%) and 23 girls (46\%); age: 10.8 months-13 years; disease duration: $5-8$ days

Treatment group: modified Ma Xing Shi Gan Tang formula plus basic therapy: Mahuang $3 \mathrm{~g}$, Xingren $6 \mathrm{~g}$,

Interventions

Jinhua 6 g, Yinhua $6 \mathrm{~g}$, Shigao $12 \mathrm{~g}$, Yuxingcao $9 \mathrm{~g}$, Banxia $6 \mathrm{~g}$, and Zhigancao $3 \mathrm{~g}$ boiled in $2 \mathrm{~L}$ water and decocted to $300 \mathrm{~mL}$; taken orally twice daily (bid)

Control group: intravenous infusion of azithromycin $(10 \mathrm{mg} / \mathrm{k} \cdot \mathrm{d})$

Outcomes

(1) Total effective rate

(1) Mortality: not mentioned; (2) relapse rate: not mentioned; (3) length of hospital stay: not mentioned; (4)

TCM outcomes, such as tongue coat and pulse condition: not mentioned; (5) economic index: not mentioned;

Notes

(6) withdrawal rates: not specified; (7) source of funding: none; (8) time to measure outcomes: not mentioned;

(9) adverse effects: not mentioned; (10) clinical recovery (e.g., cough, fever, rales, and chest films): not mentioned

\begin{tabular}{ll}
\hline & \multicolumn{1}{c}{ He et al., 2011[20] } \\
\hline & RCT: randomization mentioned, but not described in detail \\
& Allocation concealment: not mentioned \\
& Followup: not mentioned \\
& Study duration: 7 days \\
& Parallel/crossover/factorial RCT: parallel \\
& Randomization method: a telephone interview with the author revealed that a random number table was used \\
Methods & Blinding: no detailed information on blindness was offered. A telephone interview with the author revealed that \\
& blinding was used on outcome assessment \\
& ITT: not mentioned \\
& Setting: inpatients \\
& Country: China \\
& Number: 80 patients with childhood pneumonia \\
& Treatment group: 40 patients with childhood pneumonia: 22 boys (55\%) and 18 girls (45\%); mean age: $1.8 \pm 1.10$ \\
& years \\
& Control group: 40 patients with childhood pneumonia: 21 boys (52.5\%) and 19 girls (47.5\%); mean age: $1.75 \pm$ \\
& 1.151 years \\
& Disease duration: $7.50 \pm 0.50$ days \\
Participants &
\end{tabular}


TABLe 2: Continued.

\begin{tabular}{|c|c|}
\hline \multirow[t]{2}{*}{ Interventions } & $\begin{array}{l}\text { Treatment group: modified Ma Xing Shi Gan Tang formula plus basic therapy: Mahuang } 3 \mathrm{~g} \text {, Xingren } 3 \mathrm{~g} \text {, } \\
\text { Shigao } 9 \mathrm{~g} \text {, Suzi } 3 \mathrm{~g} \text {, Shangbaipi } 6 \mathrm{~g} \text {, Kuandonghua } 6 \mathrm{~g} \text {, Banxia } 6 \mathrm{~g} \text {, Tinglizi } 3 \mathrm{~g} \text {, Yuxingcao } 3 \mathrm{~g} \text {, and Gancao } 3 \mathrm{~g} \\
\text { boiled in } 2 \mathrm{~L} \text { water and decocted to } 300 \mathrm{~mL} \text {, taken orally twice daily (bid) }\end{array}$ \\
\hline & $\begin{array}{l}\text { Control group: basic therapy included supporting treatment and intravenous infusion of ceftazidime } \\
(0.1 \mathrm{~g} / \mathrm{kg} \cdot \mathrm{d})\end{array}$ \\
\hline Outcomes & (1) Total effective rate \\
\hline Notes & $\begin{array}{l}\text { (1) Mortality: not mentioned; (2) relapse rate: not mentioned; (3) length of hospital stay: not mentioned; (4) } \\
\text { TCM outcomes, such as tongue coat and pulse condition: not mentioned; (5) adverse effects: not mentioned; (6) } \\
\text { economic index: not mentioned; (7) withdrawal rates: not mentioned; (8) source of funding: none; (9) time to } \\
\text { measure outcomes: not mentioned; (10) clinical recovery (e.g., cough, fever, rales, and chest films): not } \\
\text { mentioned }\end{array}$ \\
\hline \multicolumn{2}{|r|}{ Zhang, $2012[21]$} \\
\hline \multirow{9}{*}{ Methods } & RCT: randomization mentioned, but not described in detail \\
\hline & Allocation concealment: not mentioned \\
\hline & Followup: not mentioned \\
\hline & Study duration: 5-7 days \\
\hline & Parallel/crossover/factorial RCT: parallel \\
\hline & $\begin{array}{l}\text { Randomization method: a telephone interview with the author revealed that a computer-generated } \\
\text { random-number table was used }\end{array}$ \\
\hline & $\begin{array}{l}\text { Blinding: no detailed information on blindness was offered. A telephone interview with the author revealed that } \\
\text { blinding was used on the outcome assessment }\end{array}$ \\
\hline & ITT: not mentioned \\
\hline & Setting: inpatients \\
\hline \multirow{4}{*}{ Participants } & Country: China \\
\hline & Number: 128 patients with childhood pneumonia \\
\hline & Treatment group: 64 patients with childhood pneumonia in the treatment group \\
\hline & Control group: 64 patients with childhood pneumonia in the control group \\
\hline \multirow{3}{*}{ Interventions } & In two groups, 79 boys (55\%) and 49 girls ( $45 \%$ ); age 1-14 years old; disease duration: $5-7$ days \\
\hline & $\begin{array}{l}\text { Treatment group: San Ao Tang formula plus basic therapy; Mahuang } 3 \mathrm{~g} \text {, Xingren } 12 \mathrm{~g} \text {, Gancao } 3 \mathrm{~g} \text { boiled in } 2 \mathrm{~L} \\
\text { water and decocted to } 250 \mathrm{~mL} \text {. Taken orally three times daily (tid) }\end{array}$ \\
\hline & $\begin{array}{l}\text { Control group: basic therapy included symptomatic therapy and orally azithromycin } 10 \mathrm{mg} / \mathrm{kg} \text { one time per day } \\
\text { (qd) }\end{array}$ \\
\hline Outcomes & $\begin{array}{l}\text { (1) Total effective rate; (2) clinical recovery (e.g., cough, fever, rales, and chest films); (3) adverse effects (e.g., } \\
\text { nausea) }\end{array}$ \\
\hline Notes & $\begin{array}{l}\text { (1) Mortality: not mentioned; (2) relapse rate: not mentioned; (3) length of hospital stay: not mentioned; (4) } \\
\text { TCM outcomes, such as tongue coat and pulse condition: not mentioned; (5) economic index: not mentioned; } \\
\text { (6) withdrawal rates: not mentioned; (7) source of funding: none; (8) time to measure outcomes: not mentioned }\end{array}$ \\
\hline \multicolumn{2}{|r|}{ Wang et al., 2009 [22] } \\
\hline \multirow{8}{*}{ Methods } & RCT: randomization mentioned, but not described in detail \\
\hline & Allocation concealment: not mentioned \\
\hline & Followup: not mentioned \\
\hline & Study duration: 7 days \\
\hline & Parallel/crossover/factorial RCT: parallel \\
\hline & $\begin{array}{l}\text { Randomization method: a telephone interview with the author revealed that a computer-generated } \\
\text { random-number table was used }\end{array}$ \\
\hline & $\begin{array}{l}\text { Blinding: no detailed information on blindness was offered. A telephone interview with the author revealed that } \\
\text { blinding was used on outcome assessment }\end{array}$ \\
\hline & ITT: not mentioned \\
\hline
\end{tabular}


TABLE 2: Continued.

\begin{tabular}{|c|c|}
\hline & Setting: patient source not mentioned \\
\hline \multirow{6}{*}{ Participants } & Country: China \\
\hline & Number: 200 patients with childhood pneumonia \\
\hline & Treatment group: 100 patients with childhood pneumonia in the treatment group \\
\hline & Control group: 100 patients with childhood pneumonia in the control group \\
\hline & Did not mention the number of boys and girls \\
\hline & Disease duration: $5-7$ days \\
\hline \multirow[t]{2}{*}{ Interventions } & $\begin{array}{l}\text { Treatment group: Zhi Sou San formula plus basic therapy: Jiegeng 6-9 g, Gancao 3-6 g, Ziwan 3-6 g, Chenpi } \\
\text { 3-6 g, Xingren 3-6 g, Baiguo 3-6 g, Huangqi 3-6 g, Chaomaiya 3-6 g, Yunling 3-6 g, and Baiqian 3-6 g boiled } \\
\text { in } 2 \text { L water. Taken orally three times daily (tid) }\end{array}$ \\
\hline & $\begin{array}{l}\text { Control group: basic therapy included symptomatic therapy and intravenous infusion of erythrocin } \\
(30 \mathrm{mg} / \mathrm{kg} \cdot \mathrm{d})\end{array}$ \\
\hline Outcomes & $\begin{array}{l}\text { (1) Total effective rate; (2) clinical recovery (e.g., cough, fever, and rales); (3) adverse effects (e.g., nausea, } \\
\text { vomiting, and gastrointestinal bleeding) }\end{array}$ \\
\hline Notes & $\begin{array}{l}\text { (1) Mortality: not mentioned; (2) relapse rate: not mentioned; (3) length of hospital stay: not mentioned; (4) } \\
\text { TCM outcomes, such as tongue coat and pulse condition: not mentioned; (5) economic index: not mentioned; } \\
\text { (6) withdrawal rates: not mentioned; (7) source of funding: none; (8) time to measure outcomes: not } \\
\text { mentioned; (9) chest films: not mentioned }\end{array}$ \\
\hline \multicolumn{2}{|r|}{ Lv et al., 2009 [23] } \\
\hline \multirow{8}{*}{ Methods } & RCT: randomization mentioned, but not described in detail \\
\hline & Allocation concealment: not mentioned \\
\hline & Followup was not mentioned \\
\hline & Study duration: 10 days \\
\hline & Parallel/crossover/factorial RCT: parallel \\
\hline & $\begin{array}{l}\text { Randomization method: a telephone interview with the author revealed that a random number table was used } \\
\text { to generate the random sequence }\end{array}$ \\
\hline & $\begin{array}{l}\text { Blinding: no detailed information on blindness was offered. A telephone interview with the author revealed that } \\
\text { blinding was used on the outcome assessment }\end{array}$ \\
\hline & ITT: not mentioned \\
\hline \multirow{5}{*}{ Participants } & Setting: inpatients \\
\hline & Country: China \\
\hline & Number: 60 patients with childhood pneumonia \\
\hline & $\begin{array}{l}\text { Treatment group: } 30 \text { patients with childhood pneumonia: } 17 \text { boys ( } 56.7 \%) \text { and } 13 \text { girls ( } 43.3 \%) \text {; age: } 8 \text { months-13 } \\
\text { years; disease duration: } 7-18 \text { days; mean: } 14 \text { days }\end{array}$ \\
\hline & $\begin{array}{l}\text { Control group: } 30 \text { patients with childhood pneumonia: } 16 \text { boys }(53.3 \%) \text { and } 14 \text { girls ( } 46.7 \%) \text {; age: } 7 \text { months-12 } \\
\text { years; disease duration: } 7-18 \text { days; mean: } 13.5 \text { days }\end{array}$ \\
\hline \multirow[t]{2}{*}{ Interventions } & $\begin{array}{l}\text { Treatment group: self-developed TCM prescription plus basic therapy: Mahuang } 3 \mathrm{~g} \text {, Xingren } 3 \mathrm{~g} \text {, } \\
\text { Rengongniuhuang } 3 \mathrm{~g} \text {, Bingpian } 2 \mathrm{~g} \text {, Shengshigao } 3 \mathrm{~g} \text {, Zhusha } 2 \mathrm{~g} \text {, Chuanbeimu } 2 \mathrm{~g} \text {, Huanglian } 2 \mathrm{~g} \text {, Banxia } 2 \mathrm{~g} \text {, } \\
\text { Dannanxing } 2 \mathrm{~g} \text {, Shangbaipi } 2 \mathrm{~g} \text {, Huangqin } 2 \mathrm{~g} \text {, and Gancao } 2 \mathrm{~g} \text { boiled in } 3 \mathrm{~L} \text { water and decocted to } 300 \mathrm{~mL} \text {. } \\
\text { Taken orally three times daily (tid) }\end{array}$ \\
\hline & $\begin{array}{l}\text { Control group: basic therapy included symptomatic therapy and intravenous infusion of erythrocin } \\
(20 \mathrm{mg} / \mathrm{kg} \text { bid) }\end{array}$ \\
\hline Outcomes & (1) Total effective rate; (2) clinical recovery (e.g., cough, fever, rales, and chest films) \\
\hline Notes & $\begin{array}{l}\text { (1) Mortality: not mentioned; (2) relapse rate: not mentioned; (3) length of hospital stay: not mentioned; (4) } \\
\text { TCM outcomes, such as tongue coat and pulse condition: not mentioned; (5) economic index: not mentioned; } \\
\text { (6) withdrawal rates: not mentioned; (7) source of funding: none; (8) time to measure outcomes: mentioned; (9) } \\
\text { adverse effects: not mentioned }\end{array}$ \\
\hline
\end{tabular}


TABLE 2: Continued.

\begin{tabular}{|c|c|}
\hline & Lei, $2010[24]$ \\
\hline \multirow{7}{*}{ Methods } & RCT: randomization mentioned, but not described in detail \\
\hline & Allocation concealment: not mentioned \\
\hline & Followup: not mentioned \\
\hline & Study duration: 7 days \\
\hline & Parallel/crossover/factorial RCT: parallel \\
\hline & $\begin{array}{l}\text { Randomization method: a telephone interview with the author revealed that a computer-generated } \\
\text { random-number table was used }\end{array}$ \\
\hline & $\begin{array}{l}\text { Blinding: no detailed information on blindness was offered. A telephone interview with the author revealed that } \\
\text { blinding was used on the outcome assessment }\end{array}$ \\
\hline \multirow{6}{*}{ Participants } & ITT: not mentioned \\
\hline & Setting: inpatients \\
\hline & Country: China \\
\hline & Number: 160 patients with childhood pneumonia \\
\hline & $\begin{array}{l}\text { Treatment group: } 80 \text { patients with childhood pneumonia: } 52 \text { boys ( } 65 \%) \text { and } 28 \text { girls (35\%); age: } 6 \text { months-12 } \\
\text { years; disease duration: } 2-7 \text { days }\end{array}$ \\
\hline & $\begin{array}{l}\text { Control group: } 80 \text { patients with childhood pneumonia: } 54 \text { boys (67.5\%) and } 26 \text { girls (32.5\%); age: } 5 \text { months-13 } \\
\text { years; disease duration: } 1-6 \text { days }\end{array}$ \\
\hline \multirow[t]{2}{*}{ Interventions } & $\begin{array}{l}\text { Treatment group: Tanreqing injection plus basic therapy: } 30-50 \mathrm{~mL} / \mathrm{kg} \text { Tanreqing injection }+50-100 \mathrm{~mL} 10 \% \\
\text { GS intravenous infusion once daily }(\mathrm{qd})\end{array}$ \\
\hline & $\begin{array}{l}\text { Control group: basic therapy included anti-inflammatory, symptomatic therapy. Did not provide any detailed } \\
\text { information about the anti-inflammatory, symptomatic therapy }\end{array}$ \\
\hline Outcomes & (1) Total effective rate; (2) clinical recovery (e.g., cough, fever, and rales). \\
\hline Notes & $\begin{array}{l}\text { (1) Mortality: not mentioned; (2) relapse rate: not mentioned; (3) length of hospital stay: not mentioned; (4) } \\
\text { TCM outcomes, such as tongue coat and pulse condition: not mentioned; (5) economic index: not mentioned; } \\
\text { (6) withdrawal rates: not mentioned; (7) source of funding: none; (8) time to measure outcomes: mentioned; (9) } \\
\text { chest films: not mentioned; (10) adverse effects: not mentioned }\end{array}$ \\
\hline \multicolumn{2}{|r|}{ Shi, 2009 [25] } \\
\hline \multirow{8}{*}{ Methods } & RCT: randomization mentioned, but not described in detail \\
\hline & Allocation concealment: not mentioned \\
\hline & Followup: not mentioned \\
\hline & Study duration: 14 days \\
\hline & Parallel/crossover/factorial RCT: parallel \\
\hline & Randomization method: a random number table was used to generate the random sequence \\
\hline & $\begin{array}{l}\text { Blinding: no detailed information on blindness was offered. A telephone interview with the author revealed that } \\
\text { blinding was not used on study }\end{array}$ \\
\hline & ITT: not mentioned \\
\hline \multirow{5}{*}{ Participants } & Setting: inpatients \\
\hline & Country: China \\
\hline & Number: 80 patients with childhood pneumonia \\
\hline & Treatment group: 40 patients with childhood pneumonia: 21 boys (52.5\%) and 19 girls (47.5\%) \\
\hline & $\begin{array}{l}\text { Control group: } 40 \text { patients with childhood pneumonia: } 24 \text { boys ( } 60 \%) \text { and } 16 \text { girls ( } 40 \%) \text {; age: } 5 \text { months-13 } \\
\text { years, in twogroups; disease duration: } 1-3 \text { days }\end{array}$ \\
\hline \multirow[t]{2}{*}{ Interventions } & $\begin{array}{l}\text { Treatment group: Reduning injection plus basic therapy: } 0.5-1.0 \mathrm{~mL} / \mathrm{kg} \cdot \mathrm{d} \text { Reduning injection }+250 \mathrm{~mL} 5 \% \mathrm{GS} \\
\text { intravenous infusion once daily (qd) }\end{array}$ \\
\hline & $\begin{array}{l}\text { Control group: basic therapy included symptomatic therapy and intravenous infusion of } 10 \mathrm{mg} / \mathrm{kg} \cdot \mathrm{d} \\
\text { azithromycin for } 5 \text { days,stop } 3 \text { days, then changed to oral } 10 \mathrm{mg} / \mathrm{kg} \cdot \mathrm{d} \text { azithromycin for } 3 \text { days }\end{array}$ \\
\hline Outcomes & (1) Total effective rate;(2) clinical recovery (e.g., cough, fever, rales, and chest films) \\
\hline Notes & $\begin{array}{l}\text { (1) Mortality: not mentioned; (2) relapse rate: not mentioned; (3) length of hospital stay: not mentioned; (4) } \\
\text { TCM outcomes, such as tongue coat and pulse condition: not mentioned; (5) economic index: not mentioned; } \\
\text { (6) withdrawal rates: not mentioned; (7) source of funding: none; (8) time to measure outcomes: mentioned; (9) } \\
\text { adverse effects: not mentioned }\end{array}$ \\
\hline
\end{tabular}


TABLE 2: Continued.

Pan, 2011 [26]

RCT: randomization mentioned, but not described in detail

Allocation concealment: not mentioned

Followup: not mentioned

Methods

Study duration: 7 days

Parallel/crossover/factorial RCT: parallel

Randomization method: a telephone interview with the author revealed that a random number table was used to generate the random sequence

Blinding: no detailed information on blindness was offered. A telephone interview with the author revealed thatthe outcome assessment was blinding

ITT: not mentioned.

Setting: patient source not mentioned.

Country: China

Participants

Number: 140 patients with childhood pneumonia

Treatment group: 70 patients with childhood pneumonia: 42 boys (60\%) and 28 girls (40\%); age: 1-9 years old (mean: $5.1 \pm 1.6$ years); disease duration: $2-7$ days

Control group: 70 patients with childhood pneumonia: 36 boys (51.4\%) and 34 girls (48.6\%); age: 1-10 years old (mean: $5.1 \pm 1.6$ years); disease duration: 1-7 days

Treatment group: Reduning injection plus basic therapy: $0.5-0.8 \mathrm{~mL} / \mathrm{kg}$ Reduning injection $+100 \mathrm{~mL} 5 \%$ GS

Interventions intravenous infusion once daily ( $\mathrm{qd}$ )

Control group: intravenous infusion of $10 \mathrm{mg} / \mathrm{kg} \cdot \mathrm{d}$ azithromycin $+5 \%$ GS once daily $(\mathrm{qd})$

Outcomes

(1) Total effective rate; (2) clinical recovery (e.g., cough, fever, and rales)

(1) Mortality: not mentioned; (2) relapse rate: not mentioned; (3) length of hospital stay: not mentioned; (4)

Notes

TCM outcomes, such as tongue coat and pulse condition: not mentioned; (5) economic index: not mentioned;

(6) withdrawal rates: not mentioned; (7) source of funding: mentioned; (8) time to measure outcomes: not mentioned; (9) adverse effects: not mentioned; (10) chest films: not mentioned

Duan and Feng, 2011 [27]

RCT: randomization mentioned, but not described in detail

Allocation concealment: not mentioned

Followup was not mentioned

Methods

Study duration: 14 days

Parallel/crossover/factorial RCT: parallel

Randomization method: a telephone interview with the author revealed that a random number table was used to generate the random sequence

Blinding: no detailed information on blindness was offered. A telephone interview with the author revealed that the outcome assessment was blinded

ITT: not mentioned

Setting: outpatients and inpatients

Participants

Country: China

Number: 60 patients with childhood pneumonia

In two groups: 35 boys (58.3\%) and 25 girls (41.7\%); age: 1-13 years; disease duration: $2-5$ days

Treatment group: Reduning injection plus basic therapy: 10-15 mL Reduning injection $+100 \mathrm{~mL}$ 5\% GS

Interventions intravenous infusion once daily (qd)

Control group: intravenous infusion of $10 \mathrm{mg} / \mathrm{kg} \cdot \mathrm{d}$ azithromycin $+5 \% \mathrm{GS}$ once daily (qd)

Outcomes

(1) Total effective rate; (2) clinical recovery (e.g., fever)

(1) Mortality: not mentioned; (2) relapse rate: not mentioned; (3) length of hospital stay: not mentioned; (4)

Notes

TCM outcomes, such as tongue coat and pulse condition: not mentioned; (5) economic index: not mentioned;

(6) clinical recovery (e.g., cough, rales, and chest films): not mentioned; (7) withdrawal rates: not mentioned;

(8) source of funding: none; (9) time to measure outcomes: not mentioned; (10) adverse effects: not mentioned 
TABLe 2: Continued.

\begin{tabular}{|c|c|}
\hline & Wei and Feng, 2003 [28] \\
\hline \multirow{7}{*}{ Methods } & RCT: randomization mentioned, but not described in detail \\
\hline & Allocation concealment: not mentioned \\
\hline & Followup: not mentioned \\
\hline & Study duration: 7-10 days \\
\hline & Parallel/crossover/factorial RCT: parallel \\
\hline & $\begin{array}{l}\text { Randomization method: a telephone interview with the author revealed that a computer-generated } \\
\text { random-number table was used }\end{array}$ \\
\hline & $\begin{array}{l}\text { Blinding: no detailed information on blindness was offered. A telephone interview with the author revealed that } \\
\text { blinding was not used }\end{array}$ \\
\hline \multirow{7}{*}{ Participants } & ITT: not mentioned \\
\hline & Setting: inpatients \\
\hline & Country: China \\
\hline & Number: 180 patients with childhood \\
\hline & $\begin{array}{l}\text { In two groups: } 100 \text { boys ( } 55.6 \% \text { ) and } 80 \text { girls ( } 44.4 \% \text { ); age: } 2 \text { months }-5 \text { years (mean: } 2.3 \text { years); disease duration: } \\
\text { 1-7 days }\end{array}$ \\
\hline & Treatment group: 90 patients with childhood pneumonia; 52 boys (57.8\%) and 38 girls ( $42.2 \%)$ \\
\hline & Control group: 90 patients with childhood pneumonia; 48 boys (53.3\%) and 42 girls ( $46.7 \%)$ \\
\hline \multirow[t]{2}{*}{ Interventions } & $\begin{array}{l}\text { Treatment group: Chuanhuning injection plus basic therapy: } 10 \mathrm{mg} / \mathrm{kg} \cdot \mathrm{d}+50-100 \mathrm{~mL} 10 \% \mathrm{GS} \text { or NS } \\
\text { intravenous infusion once daily }(\mathrm{qd})\end{array}$ \\
\hline & Control group: intravenous infusion of $100 \mathrm{mg} / \mathrm{kg} \cdot \mathrm{d}$ piperacillin twice daily (bid) \\
\hline Outcomes & (1) Total effective rate; (2) length of hospital stay; (3) clinical recovery (e.g., cough, fever, rales, and chest films) \\
\hline Notes & $\begin{array}{l}\text { (1) Mortality: not mentioned; (2) relapse rate: not mentioned; (3) TCM outcomes, such as tongue coat and pulse } \\
\text { condition: not mentioned; (4) economic index: not mentioned; (5) withdrawal rates: not mentioned; (6) source } \\
\text { of funding: none; (7) time to measure outcomes: not mentioned; (8) adverse effects: not mentioned }\end{array}$ \\
\hline
\end{tabular}

methods and had a high risk of performance bias or a strong detection bias.

3.3.3. Flow of Participants and Intention-to-Treat. None of the studies reported withdrawal, dropout, and/or loss during followup. The method of handling missing data regarding intention-to-treat or per-protocol analysis was not addressed.

3.3.4. Selective Reporting (Reporting Bias). No detailed evidence of selective reporting was found in any of the 14 studies [15-28]. However, we believed there is a high risk of selective reporting bias because we were unable to compare the protocol with published studies.

3.3.5. Other Potential Sources of Bias. None of the 14 studies [15-28] did not describe patient compliance. The appropriateness of the statistical analyses used was assessed, and the methods of all studies were considered appropriate. Although we conducted comprehensive searches and tried to avoid bias, we could not exclude potential publication bias because all 14 studies were published in China.

\section{Effects of Interventions}

All studies compared Chinese medicinal herbs plus basic therapy to basic therapy alone. The Chinese medicinal herb treatments included the modified Ma Xing Shi Gan Tang formula, the San Ao Tang formula, the Zhi Sou San formula, a self-developed TCM prescription, Tanreqing injection, Reduning injection, and Chuanhuning injection. Antibiotics were one of main basic therapies for Childhood Pneumonia.

4.1. Total Effective Rate. A significant increase in total effective rate was observed with Chinese medicinal herbs plus basic therapy versus basic therapy (Figure 4, analysis 1.1; RR, 1.18 ; 95\% CI, 1.11-1.26).

Subgroup five studies [15-19] compared the modified Ma Xing Shi Gan Tang formula plus azithromycin versus azithromycin and showed a significant increase in total effective rate (Figure 4; analysis 1.1 .1 of analysis 1.1; RR, 1.18; 95\% CI, 1.09-1.27).

Subgroup one study [20] compared the modified Ma Xing Shi Gan Tang formula plus ceftazidime versus ceftazidime and showed no difference in total effective rate (Figure 4; analysis 1.1.2 of analysis 1.1; RR, 1.11; 95\% CI, 0.98-1.27).

Subgroup one study [21] compared the San Ao Tang formula plus azithromycin versus azithromycin and showed no difference in total effective rate (Figure 4; analysis 1.1 .3 of analysis 1.1; RR, 0.99; 95\% CI, 0.91-1.08).

Subgroup one study [22] compared the modified Zhi Sou San formula plus erythrocin versus erythrocin and showed a significant increase in total effective rate (Figure 4; analysis 1.1.4 of analysis 1.1; RR, 1.41; 95\% CI, 1.24-1.61). 
TABLE 3: Grade quality of evidence.

Chinese medicinal herbs plus basic therapy versus basic therapy alone for childhood pneumonia

Patient or population: patients with childhood pneumonia

Settings: inpatients or outpatients

Intervention: Chinese medicinal herbs plus basic therapy versus basic therapy alone

Illustrative comparative risks $(95 \% \mathrm{CI})^{*}$

Outcomes Assumed risk Corresponding risk

Relative Number of Quality of

effect Participants evidence Comments

(95\% CI) (studies) (grade)

Control Chinese medicinal herbs plus basic therapy

versus basic therapy alone

\begin{tabular}{|c|c|c|c|c|c|c|}
\hline \multirow{4}{*}{ Total effective rate } & \multicolumn{3}{|c|}{ Study population } & \multirow{4}{*}{$\begin{array}{c}1720 \\
\text { (14 studies) }\end{array}$} & \multirow{4}{*}{$\begin{array}{l}\oplus \oplus \bigcirc \bigcirc \\
\text { Low }^{1,2}\end{array}$} & \multirow{4}{*}{ Important } \\
\hline & 779 per 1000 & $\begin{array}{l}920 \text { per } 1000 \\
(865-982)\end{array}$ & RR 1.18 & & & \\
\hline & & Moderate & $(1.11-1.26)$ & & & \\
\hline & 800 per 1000 & $\begin{array}{l}944 \text { per } 1000 \\
(888-1000)\end{array}$ & & & & \\
\hline \multirow{4}{*}{ Adverse effects } & & Study population & \multirow{4}{*}{$\begin{array}{c}\text { RR 0.39 } \\
(0.09-1.72)\end{array}$} & \multirow{4}{*}{$\begin{array}{c}434 \\
\text { (3 studies) }\end{array}$} & \multirow{4}{*}{$\begin{array}{l}\oplus \bigcirc \bigcirc \bigcirc \\
\text { Very low }\end{array}$} & \multirow{4}{*}{ Important } \\
\hline & 419 per 1000 & $\begin{array}{l}\mathbf{1 6 4} \text { per } 1000 \\
(38-721)\end{array}$ & & & & \\
\hline & & Moderate & & & & \\
\hline & 113 per 1000 & $\begin{array}{l}\mathbf{4 4} \text { per } \mathbf{1 0 0 0} \\
(10 \text { to } 194)\end{array}$ & & & & \\
\hline $\begin{array}{l}\text { Time (day) to } \\
\text { improvement of } \\
\text { cough }\end{array}$ & & $\begin{array}{l}\text { The mean time (days) to improvement in } \\
\text { cough in the intervention groups was } \\
2.18 \text { lower } \\
\text { (2.66 to } 1.71 \text { lower) }\end{array}$ & & $\begin{array}{c}1208 \\
\text { (9 studies) }\end{array}$ & $\begin{array}{l}\oplus \oplus \bigcirc \bigcirc \\
\text { Low }^{1,4}\end{array}$ & Important \\
\hline $\begin{array}{l}\text { Time (day) to } \\
\text { improvement of } \\
\text { fever }\end{array}$ & & $\begin{array}{l}\text { The mean time (days) to improvement in fever } \\
\text { in the intervention groups was } \\
2.12 \text { lower } \\
\text { ( } 2.25 \text { to } 1.98 \text { lower) }\end{array}$ & & $\begin{array}{c}1262 \\
\text { (10 studies) }\end{array}$ & $\begin{array}{l}\oplus \oplus \bigcirc \bigcirc \\
\text { Low }^{1,2}\end{array}$ & Important \\
\hline $\begin{array}{l}\text { Time (day) to } \\
\text { improvement of } \\
\text { rales }\end{array}$ & & $\begin{array}{l}\text { The mean time (days) to improvement in rales } \\
\text { in the intervention groups was } \\
\mathbf{1 . 5 3} \text { lower } \\
\text { (1.84 to } 1.23 \text { lower) }\end{array}$ & & $\begin{array}{c}1208 \\
\text { (9 studies) }\end{array}$ & $\begin{array}{c}\oplus \oplus \bigcirc \bigcirc \\
\text { Low }\end{array}$ & Important \\
\hline $\begin{array}{l}\text { Time (day) to } \\
\text { improvement in } \\
\text { chest films }\end{array}$ & & $\begin{array}{l}\text { The mean time (days) to improvement in chest } \\
\text { films in the intervention groups was } \\
\text { 3.1 lower } \\
\text { (4.11 to } 2.08 \text { lower) }\end{array}$ & & $\begin{array}{c}648 \\
\text { (5 studies) }\end{array}$ & $\begin{array}{l}\oplus \oplus \bigcirc \bigcirc \\
\text { Low }^{1,4}\end{array}$ & Important \\
\hline $\begin{array}{l}\text { Length of hospital } \\
\text { stay }\end{array}$ & & $\begin{array}{l}\text { The mean length of hospital stay in the } \\
\text { intervention groups was } \\
3 \text { lower } \\
\text { ( } 3.52 \text { to } 2.48 \text { lower) }\end{array}$ & & $\begin{array}{c}180 \\
\text { (1 study) }\end{array}$ & $\begin{array}{l}\oplus \bigcirc \bigcirc \bigcirc \\
\text { Very low }\end{array}$ & Important \\
\hline
\end{tabular}

${ }^{*}$ The basis for the assumed risk (e.g., the median control group risk across studies) is provided in the footnotes. The corresponding risk (and its $95 \%$ confidence interval) is based on the assumed risk in the comparison group and the relative effect of the intervention (and its 95\% CI). CI: confidence interval; RR: risk ratio. Grade: working group grades of evidence.

High quality: further research is very unlikely to change our confidence in the estimate of effect. Handbook description: randomized controlled trial.

Moderate quality: further research is likely to have an important impact on our confidence in the estimate of effect and may change the estimate. Cochrane Handbook description: relegation randomized controlled trial.

Low quality: further research is very likely to have an important impact on our confidence in the estimate of effect and is likely to change the estimate. Cochrane Handbook description: two or more degradation factors of randomized controlled trials.

Very low quality: we are very uncertain about the estimate. Cochrane Handbook description: more than three degradation factors of randomized controlled trials.

Reduce the evidence quality factors: methodology defect, included in the research results of the inconsistency, indirect evidence, inexactness, and publication bias.

Increase the level of evidence factor: large effect quantity, confounding factors cannot change effect quantity, or the existing concentration-response relationship.

${ }^{1}$ There is a high risk of selection bias, performance bias, and detection bias.

${ }^{2}$ Some studies showed a significant difference, but some studies showed no significant difference.

${ }^{3} \mathrm{Few}$ studies included.

${ }^{4}$ The protocol of the published studies could not be compared.

${ }^{5}$ Only one study included. 


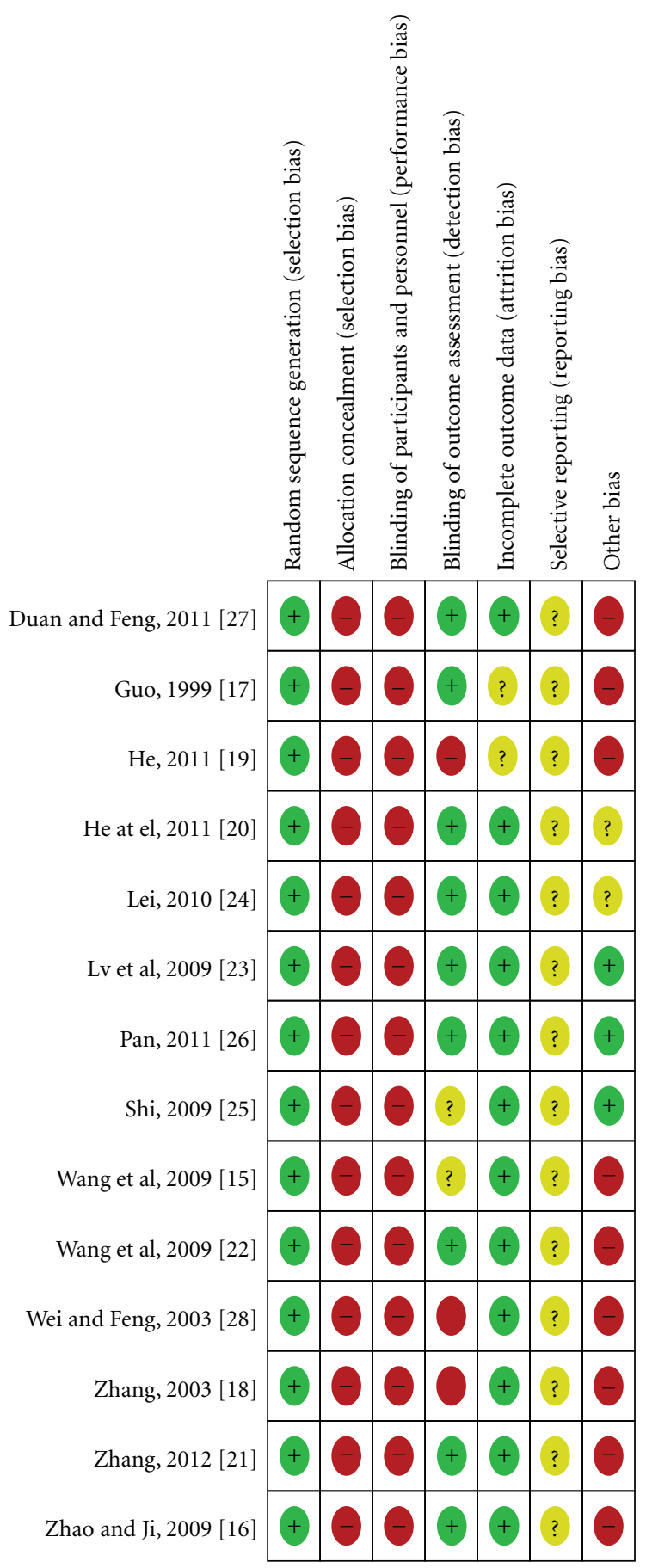

FIGURE 3: Methodological quality summary. 


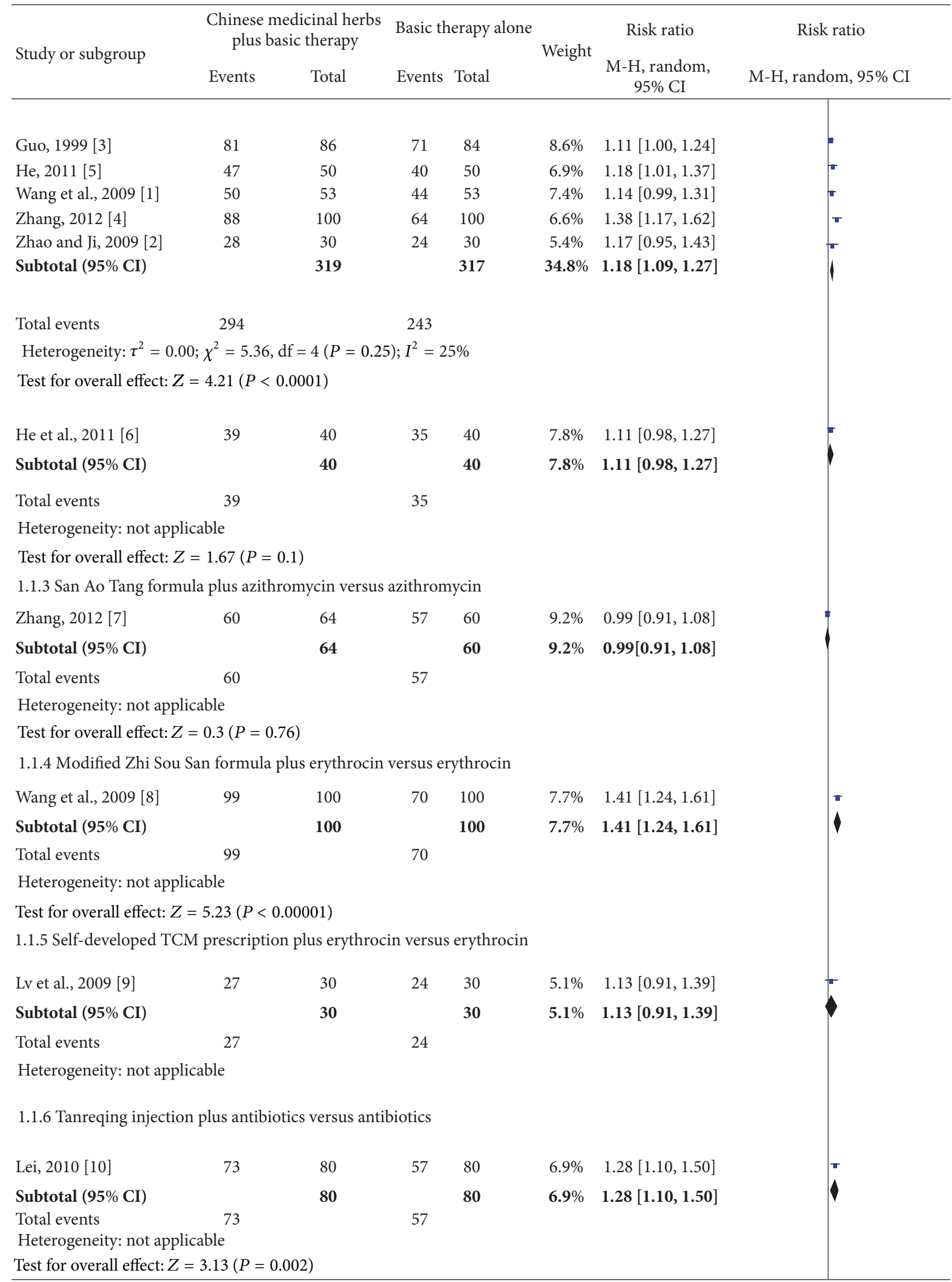




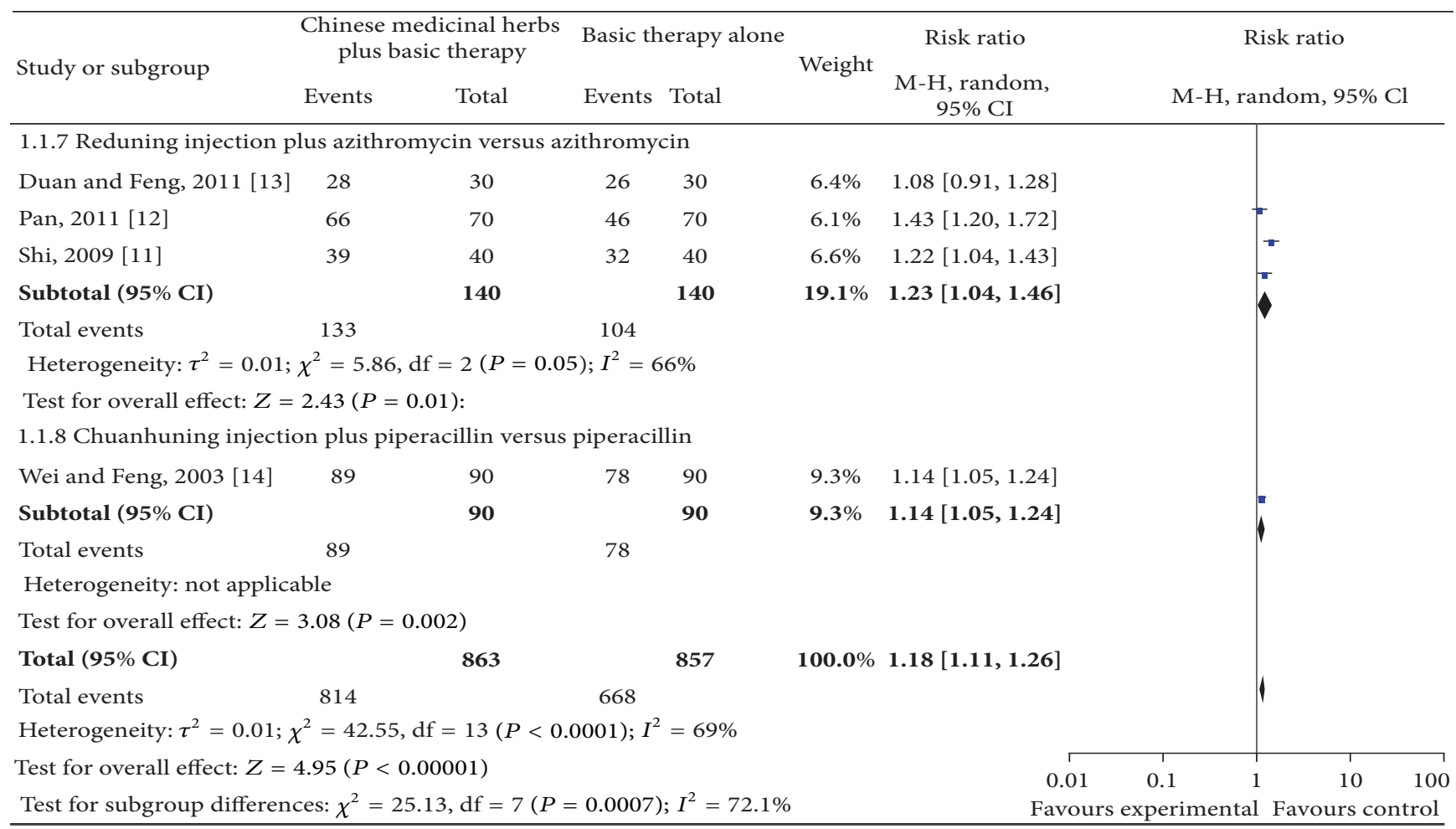

FIgURE 4: Comparison. Chinese medicinal herbs plus basic therapy versus basic therapy: outcome 1 total effective rate.

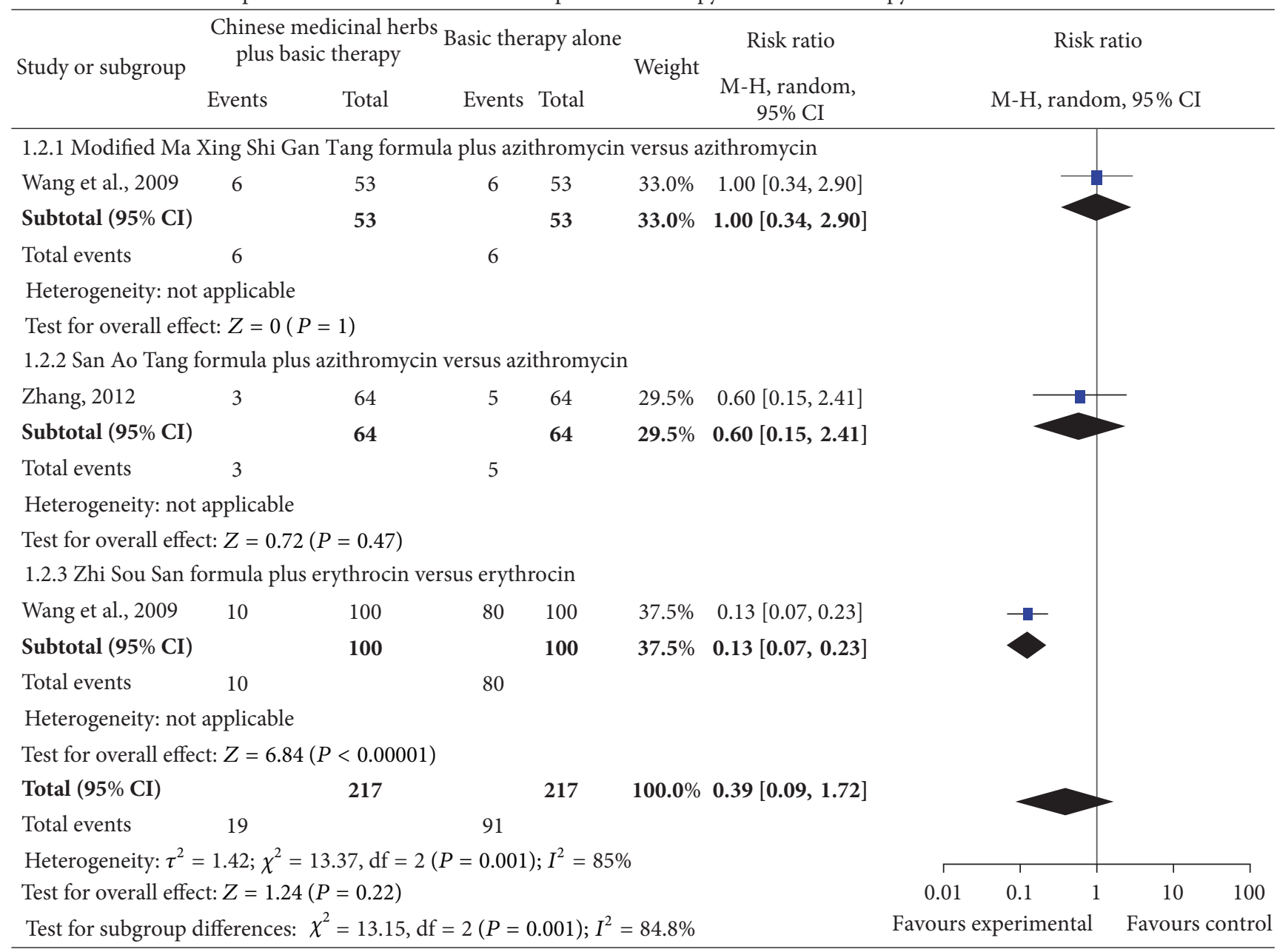




\begin{tabular}{|c|c|c|c|c|c|c|c|c|c|}
\hline \multirow{2}{*}{ Study or subgroup } & \multicolumn{3}{|c|}{$\begin{array}{l}\text { Chinese medicinal herbs } \\
\text { plus basic therapy }\end{array}$} & \multicolumn{2}{|c|}{ Basic therapy alone } & \multirow{2}{*}{ Weight } & \multirow{2}{*}{$\begin{array}{l}\text { Mean difference } \\
\text { IV, random, } \\
\text { 95\% CI }\end{array}$} & \multirow{2}{*}{\multicolumn{2}{|c|}{$\begin{array}{c}\text { Mean difference } \\
\text { IV, random, 95\% CI }\end{array}$}} \\
\hline & Mean & SD & Total & Mean SD & Total & & & & \\
\hline \multicolumn{10}{|c|}{ 1.3.1 Modified Ma Xing Shi Gan Tang formula plus azithromycin versus azithromycin } \\
\hline Zhang, 2012 & 11.04 & 3.22 & 100 & 14.224 .24 & 100 & $9.0 \%$ & $-3.18[-4.22,-2.14]$ & & \\
\hline Zhao and Ji, 2009 & 5.6 & 0.5 & 30 & $\begin{array}{ll}7.8 & 0.4\end{array}$ & 30 & $15.3 \%$ & $-2.20[-2.43,-1.97]$ & & \\
\hline Subtotal $(95 \% \mathrm{CI})$ & & & 130 & & 130 & $24.4 \%$ & $-2.55[-3.47,-1.63]$ & & \\
\hline \multicolumn{10}{|c|}{ Heterogeneity: $\tau^{2}=0.33 ; \chi^{2}=3.23, \mathrm{df}=1(P=0.07) ; I^{2}=69 \%$} \\
\hline \multicolumn{10}{|c|}{ Test for overall effect: $Z=5.43(P<0.00001)$} \\
\hline \multicolumn{10}{|c|}{ 1.3.2 San Ao Tang formula plus azithromycin versus azithromycin } \\
\hline Zhang, 2012 & 6.3 & 2.1 & 64 & $8.4 \quad 2.7$ & 64 & $10.7 \%$ & $-2.10[-2.94,-1.26]$ & 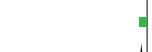 & \\
\hline Subtotal $(95 \% \mathrm{CI})$ & & & 64 & & 64 & $10.7 \%$ & $-2.10[-2.94,-1.26]$ & & \\
\hline \multicolumn{10}{|c|}{ Heterogeneity: not applicable } \\
\hline \multicolumn{10}{|c|}{ Test for overall effect: $Z=4.91(P<0.00001)$} \\
\hline \multicolumn{10}{|c|}{ 1.3.3 Modified Zhi Sou San formula plus erythrocin versus erythrocin } \\
\hline Wang et al., 2009 & 3.86 & 1.57 & 100 & $5.56 \quad 0.61$ & 100 & $14.8 \%$ & $-1.70[-2.03,-1.37]$ & 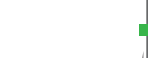 & \\
\hline Subtotal $(95 \% \mathrm{CI})$ & & & 100 & & 100 & $14.8 \%$ & $-1.70[-2.03,-1.37]$ & & \\
\hline \multicolumn{10}{|c|}{ Heterogeneity: not applicable } \\
\hline \multicolumn{10}{|c|}{ Test for overall effect: $Z=10.09(P<0.00001)$} \\
\hline \multicolumn{10}{|c|}{ 1.3.4 Self-developed TCM prescription plus erythrocin versus erythrocin } \\
\hline Lv et al., 2009 & 11.75 & 3.21 & 30 & 14.954 .12 & 30 & $4.6 \%$ & $-3.20[-5.07,-1.33]$ & $=$ & \\
\hline Subtotal (95\% CI) & & & 30 & & 30 & $4.6 \%$ & $-3.20[-5.07,-1.33]$ & 1 & \\
\hline \multicolumn{10}{|c|}{ Heterogeneity: not applicable } \\
\hline \multicolumn{10}{|c|}{ Test for overall effect: $Z=3.36(P<0.0008)$} \\
\hline \multicolumn{10}{|c|}{ 1.3.5 Tanreqing injection plus antibiotics versus antibiotics } \\
\hline Lei, 2010 & 7.02 & 0.47 & 80 & $8.22 \quad 0.63$ & 80 & $15.6 \%$ & $-1.20[-1.37,-1.03]$ & 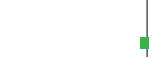 & \\
\hline Subtotal $(95 \% \mathrm{CI})$ & & & 80 & & 80 & $15.6 \%$ & $-1.20[-1.37,-1.03]$ & & \\
\hline \multicolumn{10}{|c|}{ Heterogeneity: not applicable } \\
\hline \multicolumn{10}{|c|}{ Test for overall effect: $Z=13.66(P<0.00001)$} \\
\hline \multicolumn{10}{|c|}{ 1.3.6 Reduning injection plus azithromycin versus azithromycin } \\
\hline Pan, 2011 & 4.29 & 2.16 & 70 & $6.73 \quad 2.66$ & 70 & $11.0 \%$ & $-2.44[-3.24,-1.64]$ & & \\
\hline Shi, 2009 & 7.2 & 3.9 & 40 & $9.9 \quad 4.3$ & 40 & $4.9 \%$ & $-2.70[-4.50,-0.90]$ & $\Rightarrow$ & \\
\hline Subtotal $(95 \% \mathrm{CI})$ & & & 110 & & 110 & $15.8 \%$ & $-2.48[-3.22,-1.75]$ & 1 & \\
\hline \multicolumn{10}{|c|}{ Heterogeneity: $\tau^{2}=0 ; \chi^{2}=0.07, \mathrm{df}=1(P=0.8) ; I^{2}=0 \%$} \\
\hline Test for overall effect: & $=6.64($ & $<0.00$ & & & & & & & \\
\hline 1.3.7 Chuanhuning inj & tion pl & pipera & in versu & iperacillin & & & & & \\
\hline Wei and Feng , 2003 & 4.01 & 1.21 & 90 & $6.48 \quad 1.66$ & 90 & $14.1 \%$ & $-2.47[-2.89,-2.05]$ & & \\
\hline Subtotal (95\% CI) & & & 90 & & 90 & $14.1 \%$ & $-2.47[-2.89,-2.05]$ & & \\
\hline Heterogeneity: not ap & cable & & & & & & & & \\
\hline Test for overall effect $Z$ & $=11.41$ & $<0.00$ & & & & & & & \\
\hline Total $(95 \% \mathrm{CI})$ & & & 604 & & 604 & $100.0 \%$ & $-2.18[-2.66,-1.71]$ & & \\
\hline Heterogeneity: $\tau^{2}=0$ & $; \chi^{2}=7$ & $81, \mathrm{df}=$ & $(P<0$. & $001) ; I^{2}=90$ & & & & & \\
\hline Test for overall effect: & $=8.97$ & $<0.00$ & & & & & -100 & -50 & $50 \quad 100$ \\
\hline Test for subgroup diffe & nces: $\chi^{2}$ & 50.03 & $\mathrm{f}=6(P$ & $0.00001) ; I^{2}=$ & $=88.0 \%$ & & Favours ex & erimental & Favours control \\
\hline
\end{tabular}

Figure 6: Comparison. Chinese Medicinal herbs plus basic therapy versus basic therapy: outcome 3 cough. 


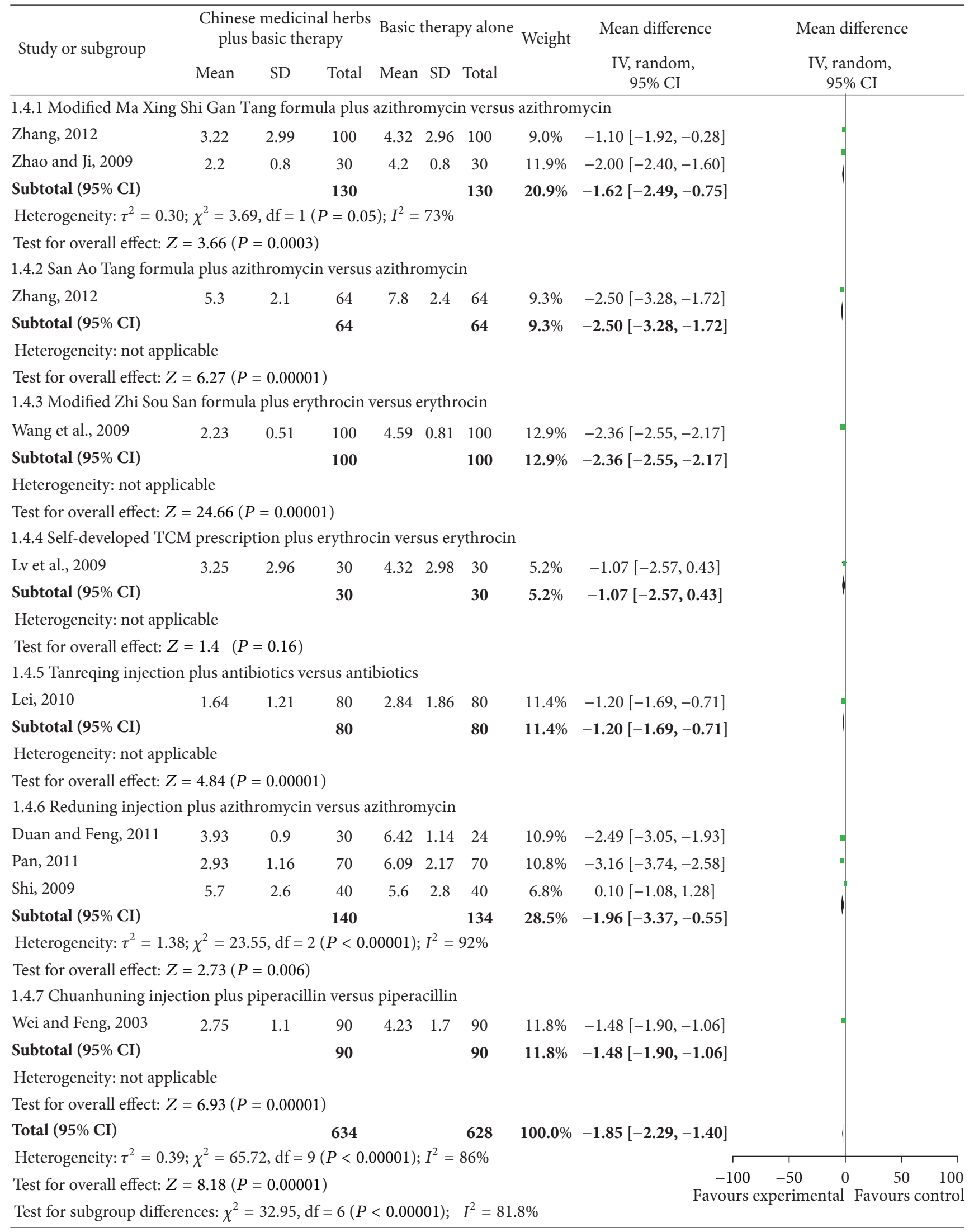




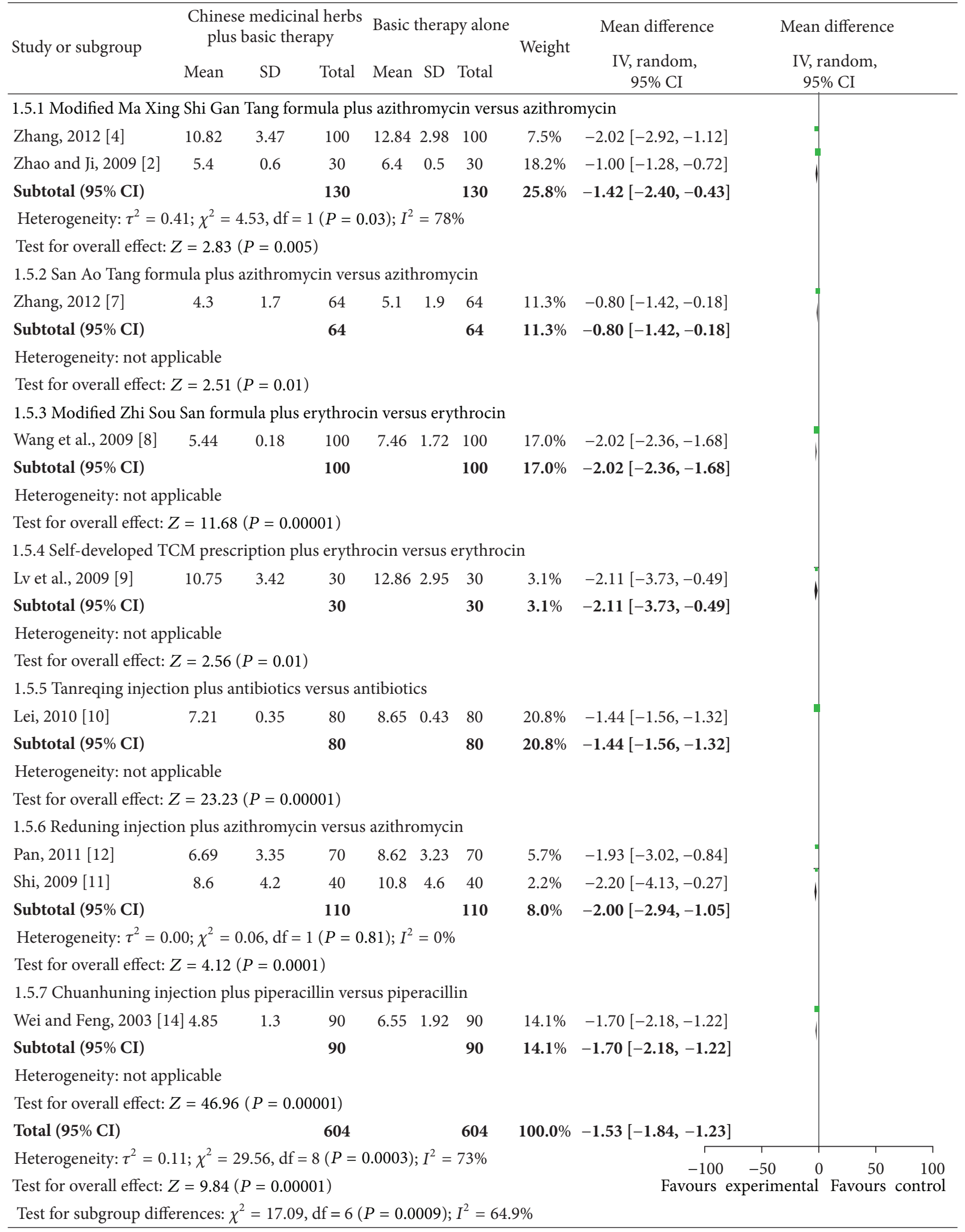

Figure 8: Comparison. Chinese medicinal herbs plus basic therapy versus basic therapy: outcome 5 rales. 


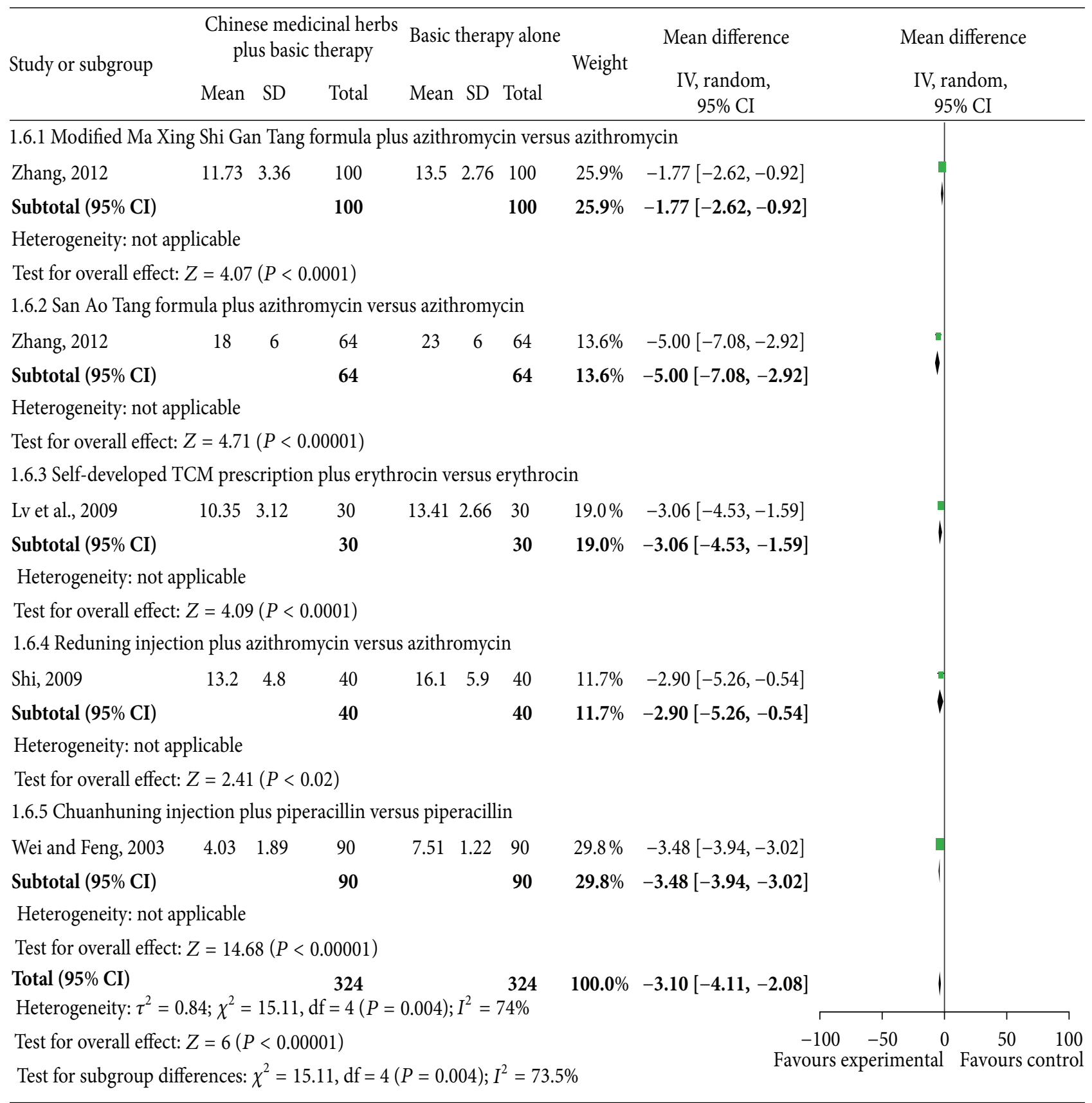

FIGURE 9: Comparison. Chinese medicinal herbs plus basic therapy versus basic therapy: outcome 6 chest films. 
Subgroup one study [23] compared a self-developed TCM prescription plus erythrocin versus erythrocin and showed no difference in total effective rate (Figure 4; analysis 1.1 .5 of analysis 1.1; RR, 1.13; 95\% CI, 0.91-1.39).

Subgroup one study [24] compared Tanreqing injection plus antibiotics versus antibiotics and showed a significant difference in total effective rate (Figure 4; analysis 1.1 .6 of analysis 1.1; RR, 1.28; 95\% CI, 1.10-1.50).

Subgroup three studies [25-27] compared Reduning injection plus azithromycin versus azithromycin and showed a significant difference in total effective rate (Figure 4; analysis 1.1.7 of analysis 1.1 ; RR, 1.23 ; 95\% CI, 1.04-1.46).

Subgroup one study [28] compared Chuanhuning injection plus piperacillin versus piperacillin and showed a significant difference in total effective rate (Figure 4; analysis 1.1.8 of analysis 1.1; RR, 1.14; 95\% CI, 1.05-1.24).

4.2. Adverse Effects. Three studies [15, 21, 22] compared Chinese medicinal herbs plus basic therapy versus basic therapy and showed no difference in adverse effects (Figure 5; analysis 1.2; RR, 0.39; 95\% Cl, 0.09-1.72).

Subgroup one study [15] compared the modified Ma Xing Shi Gan Tang formula plus azithromycin versus azithromycin and showed no difference in adverse effects (Figure 5; analysis 1.2.1 of analysis 1.2; RR, 1.00; 95\% CI, 0.34-2.90).

Subgroup one study [21] compared the San Ao Tang formula plus azithromycin versus azithromycin and showed no difference in adverse effects (Figure 5; analysis 1.2.2 of analysis 1.2; RR, 0.60; 95\% CI, 0.15-2.41).

Subgroup one study [22] compared the modified Zhi Sou San formula plus erythrocin versus erythrocin and showed a significant decrease in adverse effects (Figure 5; analysis 1.2.3 of analysis 1.2; RR, 0.13; 95\% CI, 0.07-0.23).

4.3. Time (Day) to Clinical Recovery Including Cough, Fever, Rales, and Chest Films. Studies investigating Chinese medicinal herbs plus basic therapy versus basic therapy showed a significant difference in cough (Figure 6; analysis 1.3; total $\mathrm{MD},-2.18$; 95\% Cl, (-2.66)-(-1.71)), fever (Figure 7; analysis 1.4; total MD, -1.85 ; 95\% Cl, (-2.29) $-(-1.40)$ ), rales (Figure 8; analysis 1.5 ; total $\mathrm{MD},-1.53$; $95 \% \mathrm{Cl},(-1.84)-(-1.23))$, and chest films (Figure 9; analysis 1.6; total MD, $-3.10 ; 95 \% \mathrm{Cl}$, $(-4.11)-(-2.08))$.

Subgroup two studies $[16,18]$ compared the modified Ma Xing Shi Gan Tang formula plus azithromycin versus azithromycin and showed a significant difference for cough (Figure 6; analysis 1.3 .1 of analysis 1.3; $\mathrm{MD},-2.55 ; 95 \% \mathrm{Cl}$, $(-3.47)-(-1.63)$ ), fever (Figure 7; analysis 1.4 .1 of analysis 1.4 ; $\mathrm{MD},-1.62 ; 95 \% \mathrm{Cl},(-2.49)-(-0.75)$ ), and rales (Figure 8; analysis 1.5 .1 of analysis 1.5 ; $\mathrm{MD},-1.42 ; 95 \% \mathrm{Cl},(-2.40)-$ $(-0.43))$. Subgroup one study [18] compared the modified Ma Xing Shi Gan Tang formula plus azithromycin versus azithromycin and showed a significant difference on chest films (Figure 9; analysis 1.6.1 of analysis 1.6; MD, -1.77 ; 95\% $\mathrm{Cl},(-2.62)-(-0.92))$.

Subgroup one study [21] compared the San Ao Tang formula plus azithromycin versus azithromycin and showed a significant difference in cough (Figure 6; analysis 1.3.2 of analysis $1.3 ; \mathrm{MD},-2.10 ; 95 \% \mathrm{Cl},(-2.94)-(-1.26))$, fever (Figure 7; analysis 1.4 .2 of analysis $1.4 ; \mathrm{MD},-2.50 ; 95 \% \mathrm{Cl}$, $(-3.28)-(-1.72)$ ), rales (Figure 8 ; analysis 1.5 .2 of analysis 1.5 ; $\mathrm{MD},-0.80 ; 95 \% \mathrm{Cl},(-1.42)-(-0.18))$, and chest films (Figure 9; analysis 1.6 .2 of analysis $1.6 ; \mathrm{MD},-5.00 ; 95 \% \mathrm{Cl}$, $(-7.08)-(-2.92))$.

Subgroup one study [22] compared the modified Zhi Sou San formula plus erythrocin versus erythrocin and showed a significant difference in cough (Figure 6; analysis 1.3.3 of analysis 1.3 ; MD, -1.70 ; 95\% CI, $(-2.03)-(-1.37)$ ), fever (Figure 7 analysis 1.4 .3 of analysis $1.4 ; \mathrm{MD},-2.36 ; 95 \% \mathrm{Cl}$, $(-2.55)-(-2.17)$ ), and rales (Figure 8 analysis 1.5 .3 of analysis 1.5 ; $\mathrm{MD},-2.02$; $95 \% \mathrm{Cl},(-2.36)-(-1.68))$.

Subgroup one study [23] compared a self-developed TCM prescription plus erythrocin versus erythrocin and showed a significant difference in cough (Figure 6 analysis 1.3.4 of analysis 1.3 ; MD, 3.20; 95\% CI, (-5.07)-(-1.33)), rales (Figure 8 analysis 1.5 .4 of analysis $1.5 ; \mathrm{MD},-2.11 ; 95 \% \mathrm{Cl}$, $(-3.73)-(-0.49))$, and chest films (Figure 9 analysis 1.6 .3 of analysis 1.6; $\mathrm{MD},-3.06 ; 95 \% \mathrm{Cl},(-4.53)-(-1.59))$, but no difference in fever (Figure 7 analysis 1.4 .4 of analysis 1.4; MD, -1.07 ; 95\% Cl, -2.57-0.43).

Subgroup one study [24] compared Tanreqing injection plus antibiotics versus antibiotics and showed a significant difference in cough (Figure 6 analysis 1.3 .5 of analysis 1.3; MD, -1.20 ; 95\% CI, (-1.37)-(-1.03)), fever (Figure 7 analysis 1.4 .5 of analysis $1.4 ; \mathrm{MD},-1.20 ; 95 \% \mathrm{Cl},(-1.69)-(-0.71))$, and rales (Figure 8 analysis 1.5 .5 of analysis $1.5 ; \mathrm{MD},-1.44 ; 95 \% \mathrm{Cl}$, $(-1.56)-(-1.32))$.

Subgroup two studies $[25,26]$ compared Reduning injection plus azithromycin versus azithromycin and showed a significant difference in cough (Figure 6 analysis 1.3.6 of analysis 1.3 ; MD, $-2.48 ; 95 \% \mathrm{CI},(-3.22)-(-1.75))$ and rales (Figure 8 analysis 1.5 .6 of analysis 1.5 ; $\mathrm{MD},-2.00$; 95\% CI, (-2.94)-(-1.05)). Subgroup three studies [25-27] compared Reduning injection plus azithromycin versus azithromycin and showed a significant difference in fever (Figure 7 analysis 1.4 .6 of analysis 1.4 ; MD, -1.96 ; 95\% CI, (-3.37)-(-0.55)). Subgroup one study [25] compared Reduning injection plus azithromycin versus azithromycin and showed a significant difference on chest films (Figure 9 analysis 1.6 .4 of analysis 1.6 ; $\mathrm{MD},-2.90$; 95\% CI, (-5.26)$(-0.54))$.

Subgroup one study [28] compared Chuanhuning injection plus piperacillin versus piperacillin and showed a significant difference in cough (Figure 6 analysis 1.3.7 of analysis 1.3; MD, -2.47; 95\% CI, (-2.89)-(-2.05)), fever (Figure 7 analysis 1.4 .7 of analysis 1.4 ; MD, -1.48 ; 95\% CI, (-1.90)-(-1.06)), rales (Figure 8 analysis 1.5 .7 of analysis 1.5 ; MD, -1.70 ; 95\% CI, $(-2.18)-(-1.22)$ ), and chest films (Figure 9 analysis 1.6 .5 of analysis 1.6; $\mathrm{MD},-3.48$; 95\% CI, (-3.94) $-(-3.02))$.

4.4. Length of Hospital Stay. One study [28] compared Chuanhuning injection plus piperacillin versus piperacillin and showed a significant difference in length of hospital stay (Figure 10 analysis 1.7 ; total $\mathrm{MD},-3.00$; 95\% CI, (-3.52)$(-2.48))$. 


\begin{tabular}{|c|c|c|c|c|c|c|c|c|c|c|c|}
\hline \multirow[t]{2}{*}{ Study or subgroup } & \multicolumn{3}{|c|}{$\begin{array}{l}\text { Chinese medicinal herbs } \\
\text { plus basic therapy }\end{array}$} & \multicolumn{3}{|c|}{ Basic therapy alone } & \multirow[t]{2}{*}{ Weight } & Mean difference & \multirow{2}{*}{\multicolumn{3}{|c|}{$\begin{array}{l}\text { Mean difference } \\
\text { IV, fixed, } 95 \% \text { CI }\end{array}$}} \\
\hline & Mean & $\mathrm{SD}$ & Total & Mean & SD & Total & & IV, fixed, $95 \%$ CI & & & \\
\hline \multicolumn{12}{|c|}{ 1.7.1 Chuanhuning injection plus piperacillin versus piperacillin } \\
\hline Wei and Feng, 2003 & 6.2 & 1.71 & 90 & 9.2 & 1.85 & 90 & $100.0 \%$ & $-3.00[-3.52,-2.48]$ & & & \\
\hline Subtotal (95\% CI) & & & 90 & & & 90 & $100.0 \%$ & $-3.00[-3.52,-2.48]$ & & & \\
\hline \multicolumn{12}{|c|}{ Heterogeneity: not applicable } \\
\hline \multicolumn{12}{|c|}{ Test for overall effect: $Z=11.30(P<0.00001)$} \\
\hline Total $(95 \% \mathrm{CI})$ & & & 90 & & & 90 & $100.0 \%$ & $-3.00[-3.52,-2.48]$ & 1 & & \\
\hline \multicolumn{12}{|c|}{ Heterogeneity: not applicable } \\
\hline \multicolumn{8}{|c|}{ Test for overall effect: $Z=11.30(P<0.00001)$} & -100 & $-50 \quad 0$ & 50 & 100 \\
\hline \multicolumn{8}{|c|}{ Test for subgroup differences: not applicable } & Favours & xperimental & Favours & ontrol \\
\hline
\end{tabular}

FIgURE 10: Comparison. Chinese medicinal herbs plus basic therapy versus basic therapy: outcome 7 length of hospital stay.

4.5. Other Outcomes. Mortality, relapse rate, TCM outcomes (e.g., tongue coat and pulse condition), and economic index were not reported in any of the 14 studies.

\section{GRADE Quality of Evidence}

The "GRADEprofiler" of the Cochrane Collaboration Network was used to classify the systematic review results. The quality of evidence was low to very low (Table 3).

\section{Discussion}

Based on the 14 [15-28] RCTs conducted in China, Chinese medicinal herbs increased total effective rates (e.g., ratio of signs and symptoms improvement or recovery) and improved clinical symptoms and signs (e.g., cough, fever, rales, and chest films). However, the evidence that Chinese medicinal herbs decreased adverse effects, mortality, or improved TCM outcomes (e.g., tongue coat and pulse condition) was insufficient. The quality of the evidence was weak due to selective bias, measurement bias, selective reporting bias, and imprecision. Therefore, evidence from the included studies was not enough to make any recommendations.

First, randomization was mentioned in all 14 studies. However, one study [25] described the randomization method in detail, whereas 13 did not [15-24, 26-28]. We interviewed the authors by telephone and determined that a random number table or a computer-generated randomnumber table was used to generate the allocation sequence. Second, none of the studies mentioned a blinding method, but we interviewed the authors by telephone and found that 10 studies [15-17, 20-24, 26, 27] used single blinding (i.e., the outcome assessment was blinded), and four $[18,19$, $25,28]$ did not. The lack of blinding participants, healthcare providers, and assessors can introduce performance and detection bias. Third, none of the studies addressed the incomplete outcome data, such as missing data due to attrition or exclusion. The inadequate handling of missing data can compromise statistical analyses. Fourth, the majority of experimental Chinese herbal medicine interventions were prepared by the investigators without detailed information describing their underlying rationale for the formulation, dosage, or the manufacturing process, and the quality control processes for their tested interventions are unknown. Thus, independent validation of these findings is necessary.

This paper has several methodological limitations. First, although all data were collected from reports or from direct contact with the authors, many items on the "risk of bias" assessment tool could only be classified as "unclear." Second, different Chinese herbal medicine interventions were grouped together for analysis in some cases. The results might have been compromised by the heterogeneity within each Chinese herbal medicine intervention and the study design. Third, the concept of TCM syndrome was not considered when analyzing the data, as all studies only considered Childhood Pneumonia, not TCM symptoms. Therefore, the actual therapeutic effect might not have been fully captured.

Based on these reasons, the TCM RCTs should be conducted in accordance with the Consolidated Standards of Reporting Trials for Traditional Chinese Medicine [29] detailed report.

\section{Conclusions}

Chinese herbal medicines may increase total effective rates, improve clinical symptoms and signs, and shorten the length of hospital stay for children with pneumonia. In a word, Chinese herbal medicines are effective for Childhood Pneumonia. However, there is insufficient evidence to confirm whether Chinese herbal medicines decrease adverse effects, mortality, and TCM outcomes (such as tongue coat and pulse condition). All results were supported by poor methodological quality studies. Thus, larger, multicenter, high methodological quality studies of Chinese herbal medicines for Childhood Pneumonia are needed. These studies should include patients with Childhood Pneumonia and interventions with Chinese herbal medicines. More data, particularly concerning adverse events, are necessary. Meanwhile, future studies should determine the most appropriate drug and 
dosage for Childhood Pneumonia. So, further trials would help to clarify the validity of the findings of this paper and could determine more clearly the role of Chinese herbal medicines in Childhood Pneumonia in comparison with other therapies. Consequently, the purpose is to guide our application in clinical.

\section{Conflict of Interests}

The authors declare no conflict of interests.

\section{Acknowledgments}

The authors thank the Department of Nephrology Laboratory, Guangdong Province Hospital of Chinese Medicine. The authors thank the Department of Nephropathy Medicine, Guangdong Province Hospital Of Chinese Medicine. The project was support by the Scientific Research Project of Public Welfare Industry, State Administration of Traditional Chinese Medicine of China (no. 200707004).

\section{References}

[1] A. L. Cohen, T. B. Hyde, J. Verani et al., "Integrating pneumonia prevention and treatment interventions with immunization services in resource-poor countries," Bulletin of the World Health Organization, vol. 90, pp. 289-294, 2012.

[2] J. Anthony, G. Scott, C. Wonodi, J. C. Moisi et al., "The definition of pneumonia, the assessment of severity, and clinical standardization in the pneumonia etiology research for child health study," Clinical Infectious Diseases, vol. 54, no. S2, pp. S109-S116, 2012.

[3] M. Mathisen, T. A. Strand, B. N. Sharma et al., "RNA viruses in community-acquired childhood pneumonia in semi-urban Nepal; a cross-sectional study," BMC Medicine, vol. 7, article no. 35, 2009.

[4] I. C. Michelow, K. Olsen, J. Lozano et al., "Epidemiology and clinical characteristics of community-acquired pneumonia in hospitalized children," Pediatrics, vol. 113, no. 4 I, pp. 701-707.e7, 2004.

[5] S. Tafuri, D. Martinelli, A. Grimaldi et al., "23-valent pneumococcal vaccine failure in a patient who developed pneumonia: a case report," The New Microbiologica, vol. 34, pp. 417-420, 2011.

[6] United Nations Children's Fund, Pneumonia: the Forgotten Killer of Children, United Nations Children's Fund, New York, NY, USA, 2006.

[7] G. M. Zhao, S. Black, H. Shinefield, and J. Eskola, "Rates of hospitalization and mortality for pneumonia and respiratory illness in China," in Proceedings of the 5th International Symposium on Pneumococci and Pneumococcal Diseases, 2006, PO2.08.

[8] I. Rudan, C. Boschi-Pinto, Z. Biloglav, K. Mulholland, and H. Campbell, "Epidemiology and etiology of childhood pneumonia," Bulletin of the World Health Organization, vol. 86, no. 5, pp. 408-416, 2008.

[9] The WHO Young Infant Study Group, "Bacterial etiology of serious infections in young infants in developing countries: results of multicentre study," Pediatric Infectious Diseases Journal, vol. 18, pp. 17-22, 1999.

[10] M. Korppi, O. Kiekara, T. Heiskanen-Kosma, and S. Soimakallio, "Comparison of radiological findings and microbial aetiology of childhood pneumonia," Acta Paediatrica, International Journal of Paediatrics, vol. 82, no. 4, pp. 360-363, 1993.

[11] A. L. Hersh, D. J. Shapiro, J. G. Newland, P. M. Polgreen, S. E. Beekmann, and S. S. Shah, "Variability in pediatric infectious disease consultants' recommendations for management of community-acquired pneumonia," PLoS ONE, vol. 6, no. 5, Article ID e20325, pp. 1-6, 2011.

[12] S. K. Kabra, R. Lodha, and R. M. Pandey, "Antibiotics for community acquired pneumonia inchildren," Cochrane Database of Systematic Reviews. In press.

[13] T. Wu, J. Zhang, Y. Qiu, L. Xie, and G. J. Liu, "Chinese medicinal herbs for the common cold," Cochrane Database of Systematic Reviews, no. 1, Article ID CD004782, 2007.

[14] X. Li, Y. P. Zhang, and J. B. Lou, "Ma Xing Shi Gan Tang to treat respiratory disease research progress," Journal of Guiyang College of Traditional Chinese Medicine, vol. 32, no. 5, pp. 66-68, 2010.

[15] H. M. Wang, J. L. Ge, and D. Mo, "Azithromycin combined with traditional Chinese Ma Xing Shi Gan Tang for children pneumonia and mycoplasma in 106 cases," Medical Journal of Chinese People's Health, vol. 21, no. 19, pp. 2387-2389, 2009.

[16] X. C. Zhao and W. Y. Ji, "Maxing Shigan decoction combined with azithromycin for children pneumonia clinical observation," Chinese Journal of Drug Abuse Prevention and Treatment, vol. 15, no. 6, pp. 358-360, 2009.

[17] X. H. Guo, "Integration of traditional Chinese and Western medicine for Children Pneumonia on 86 cases," Fujian Medical Journal, vol. 21, no. 2, article 90, 1999.

[18] Y. M. Zhang, "San Ao Tang auxiliary treatment for children mycoplasma pneumonia infection observation of curative effect," Chinese Traditional and Herbal Drugs, vol. 43, no. 2, pp. 341-342, 2012.

[19] D. J. He, "Combination of traditional Chinese and Western medicine for Childhood pneumonia in observation of clinical effect," Journal China Health Monthly, vol. 30, no. 7, pp. 10-11, 2011.

[20] G. P. He, H. X. Lei, and H. H. Du, "Combination of traditional Chinese and Western medicine for 40 cases of pneumonia cough," Journal of Shaanxi College of Traditional Chinese Medicine, vol. 34, no. 6, pp. 36-37, 2011.

[21] Y. M. Zhang, "San Ao Tang auxiliary treatment for children mycoplasma pneumonia infection observation of curative effect," Chinese Traditional and Herbal Drugs, vol. 43, no. 2, pp. 341-342, 2012.

[22] S. Y. Wang, W. D. Pang, Q. Y. Chu et al., "Modified Zhi Sou San combined with erythromycin in treatment of Mycoplasma pneumonia curative effect analysis," Modern Journal of Integrated Traditional Chinese and Western Medicine, vol. 18, no. 23, pp. 2797-2798, 2009.

[23] S. Q. Lv, D. Xie, S. F. Xia et al., "Combination of traditional Chinese and Western medicine for childhood mycoplasma pneumonia clinical practice," Chinese Journal of Maternal and Child Health Care, vol. 24, no. 22, pp. 3093-3094, 2009.

[24] Z. Lei, "Combination of traditional Chinese and Western medicine for 80 cases childhood pneumonia clinical observation," Journal of Traditional Chinese Medicine Information, vol. 2, no. 1, article 91, 2010.

[25] Y. F. Shi, "Combination of traditional Chinese and Western medicine for childhood mycoplasma pneumonia in 40 cases," Chinese Pediatrics of Integrated Traditional and Western Medicine, vol. 1, no. 4, pp. 364-365, 2009. 
[26] W. Q. Pan, "Combined medication for community acquired pneumonia in children 70 cases," Chinese Medicine Modern Distance Education of China, vol. 9, no. 20, pp. 56-57, 2011.

[27] X. Z. Duan and X. C. Feng, "Reduning Injection for childhood Mycoplasma pneumoniae on clinical study," Journal of Changchun University of Traditional Chinese Medicine, vol. 27, no. 2, pp. 171-172, 2011.

[28] H. Wei and S. K. Feng, "Observation on effect of Chuanhuning Injection for childhood pneumonia," Journal of Youjiang Medical College for Nationalities, vol. 25, no. 4, article 516, 2003.

[29] T. X. Wu, H. C. Shang, and Z. X. Bian, "Randomized controlled pragmatic trial: concept, design, practice," Chinese Journal of Evidence-Based Medicine, vol. 9, no. 12, pp. 1277-1280, 2009. 


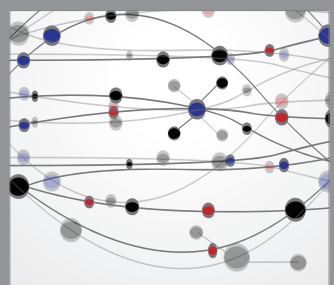

The Scientific World Journal
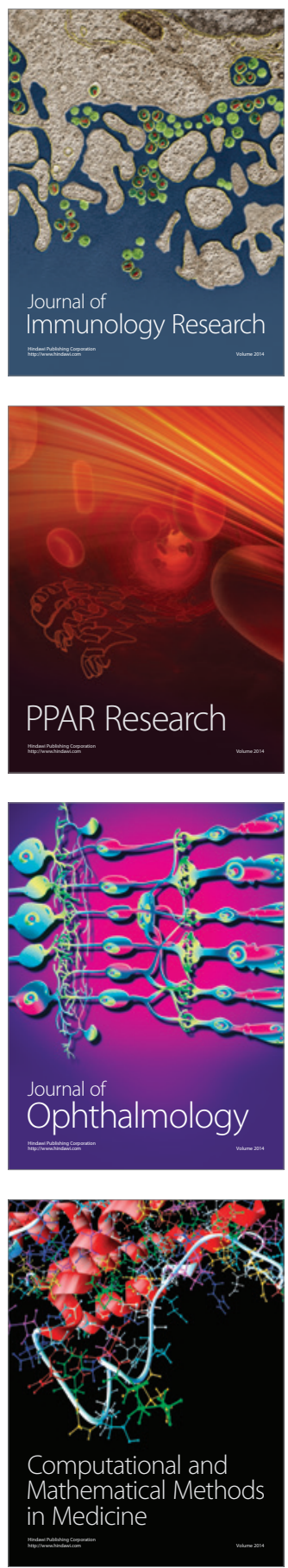

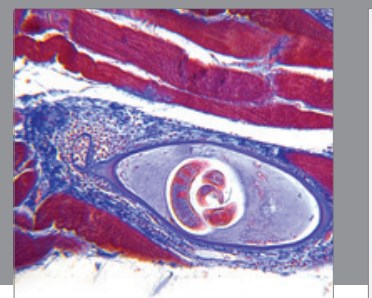

Gastroenterology

Research and Practice
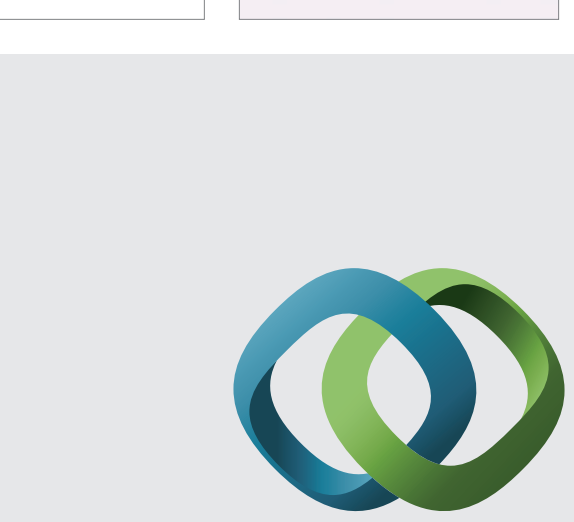

\section{Hindawi}

Submit your manuscripts at

http://www.hindawi.com
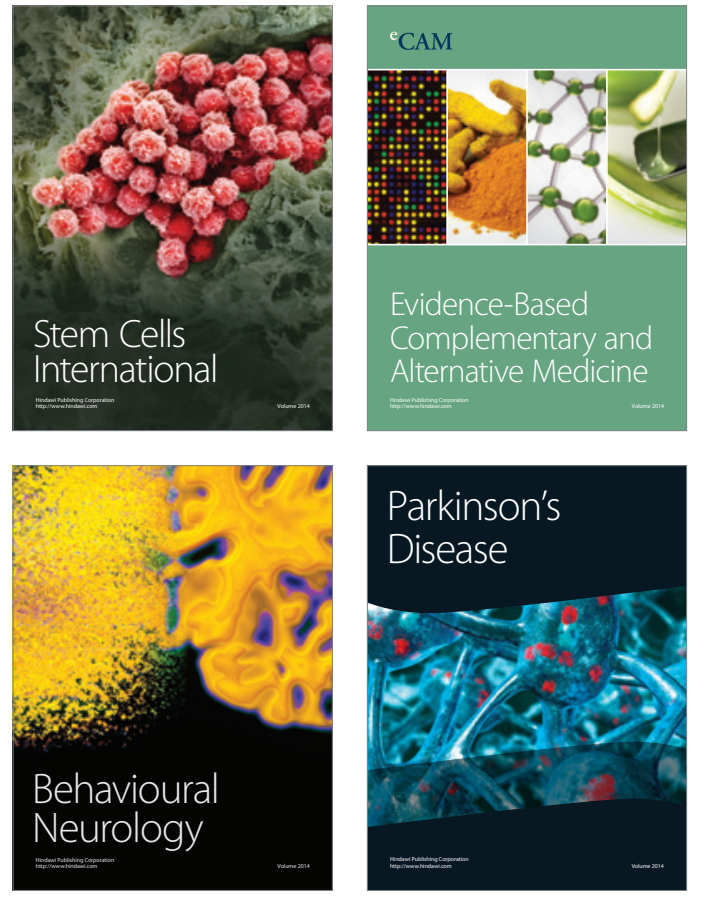
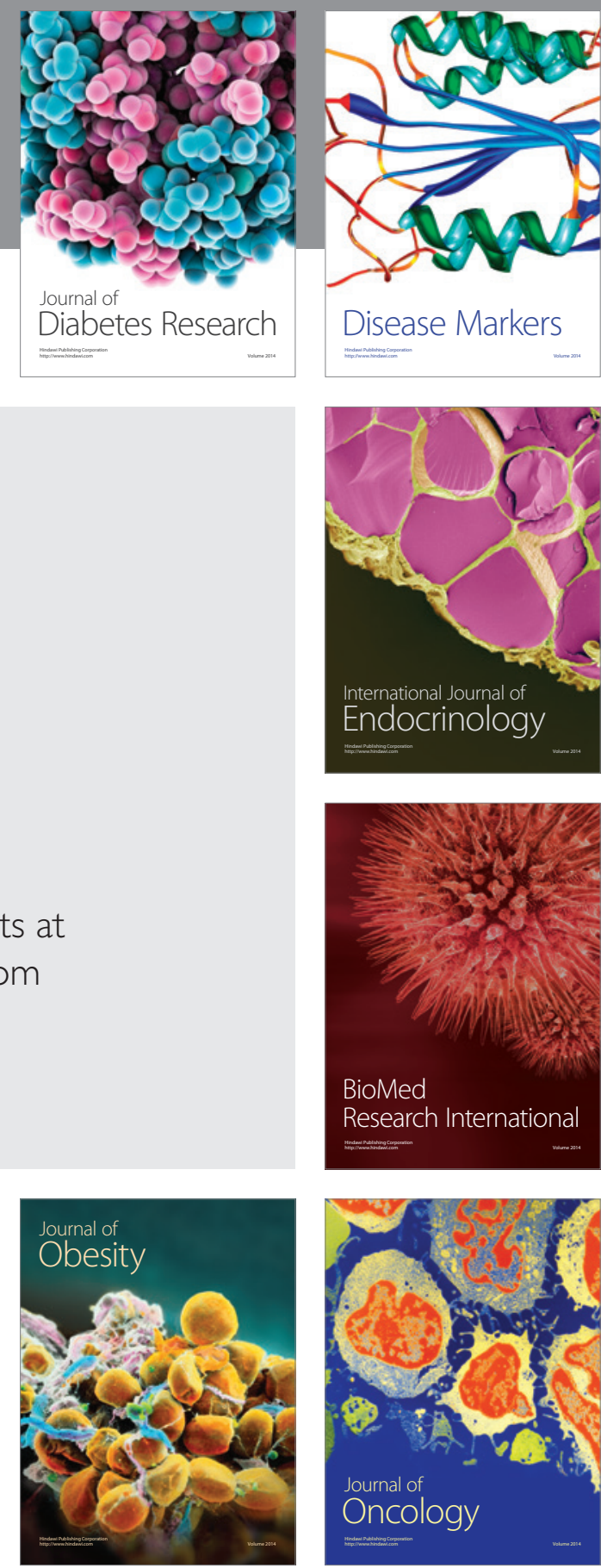

Disease Markers
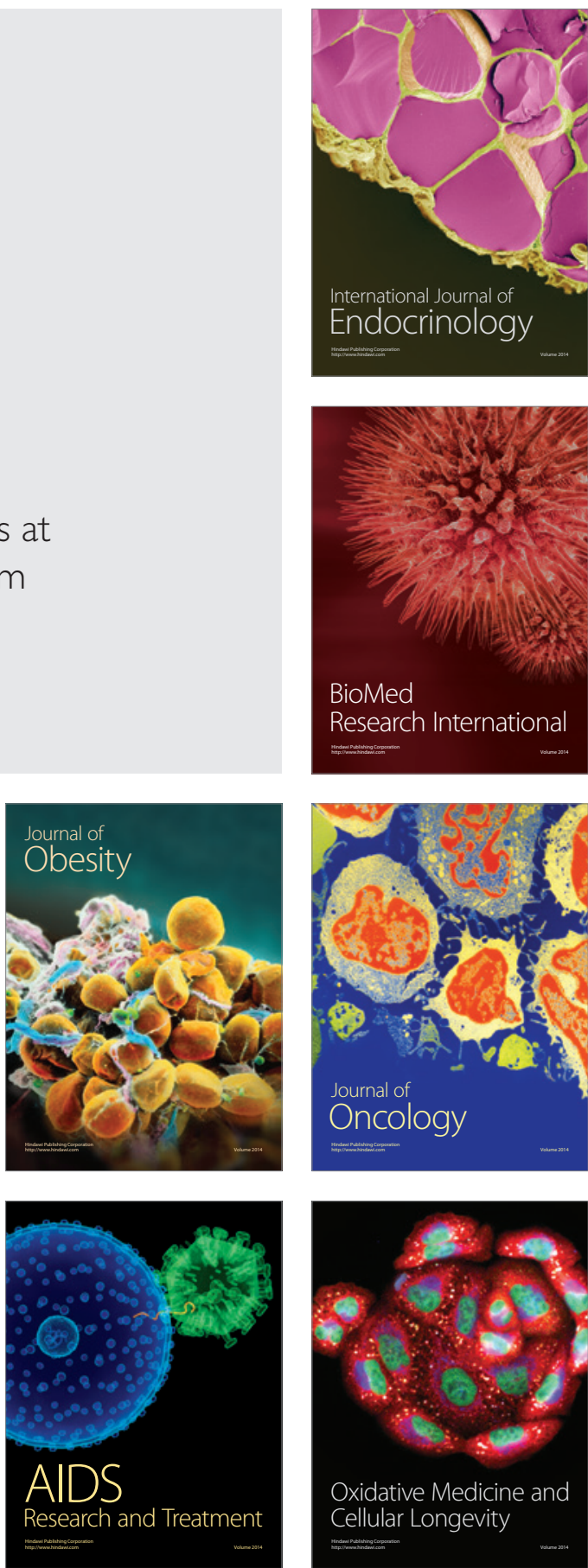